NBER WORKING PAPER SERIES

\title{
INTERTEMPORAL DISTORTIONS IN THE SECOND BEST
}

\author{
Stefania Albanesi \\ Roc Armenter \\ Working Paper 13629 \\ http://www.nber.org/papers/w13629
}

\author{
NATIONAL BUREAU OF ECONOMIC RESEARCH \\ 1050 Massachusetts Avenue \\ Cambridge, MA 02138
}

November 2007

We would like to thank Fernando Alvarez, Manuel Amador, Boragan Aruoba, Marco Bassetto, Martin Bodenstein, V.V. Chari, Sanjay Chugh, Dirk Krueger, Harald Uhlig, Pierre Yared, three anonymous referees, and seminar participants at the University of Maryland, the University of Pennsylvania, Georgetown, Stanford, Chicago GSB, Duke, Cornell, UCSB, the University of Cambridge, the Federal Reserve Banks of New York, Philadelphia and San Francisco, and the NBER Summer Institute for helpful comments and suggestions. The views expressed here do not necessarily reflect the views of the Federal Reserve Bank of New York, the Federal Reserve System, or the National Bureau of Economic Research.

(C) 2007 by Stefania Albanesi and Roc Armenter. All rights reserved. Short sections of text, not to exceed two paragraphs, may be quoted without explicit permission provided that full credit, including (C notice, is given to the source. 
Intertemporal Distortions in the Second best

Stefania Albanesi and Roc Armenter

NBER Working Paper No. 13629

November 2007, Revised June 2009

JEL No. E6,H21

\begin{abstract}
$\underline{\text { ABSTRACT }}$
This paper studies the long run properties of intertemporal distortions in a broad class of second best economies. Our unified framework encompasses and extends many well known models, such as variants of the Ramsey taxation model with aggregate or idiosyncratic risk, and economies with incentive compatibility constraints due to limited commitment, political economy, self-enforcement or private information, or combinations of these. We identify a sufficient condition that rules out permanent intertemporal distortions: If there exists an allocation that satisfies all constraints and eventually converges to the limiting first best allocation, then intertemporal distortions are temporary in the second best. This result uncovers a common optimality principle linking the intertemporal allocation of resources with the ability to frontload distortions for this broad class of environments. A series of applications illustrates the significance of these findings.
\end{abstract}

Stefania Albanesi

Columbia University

1022 International Affairs Building

420 West 118th Street

New York, NY 10027

and NBER

sa2310@columbia.edu

Roc Armenter

Research Department

Federal Reserve Bank of Philadelphia

Ten Independence Hall

Philadelphia, PA 19106-1574

roc.armenter@phil.frb.org 


\section{Introduction}

The intertemporal allocation of resources has implications for aggregate income, risk sharing, asset pricing, and other key areas of interest in macroeconomics. If feasibility were the only constraint, then it would be possible to attain a fully efficient - first best - allocation. In reality, a variety of additional constraints imply that the allocation cannot be fully efficient and will feature distortions. This paper studies the long run behavior of intertemporal distortions in a broad class of constrainedefficient - second best - economies.

Many possible sources of frictions can constrain the allocation of resources. Perhaps the most immediate of these is taxes. The Ramsey model provides a benchmark for optimal taxation in macroeconomics. Chamley (1986) and Judd (1985) first established that capital income taxes are zero in the steady state and all intertemporal distortions eventually vanish, a finding that has been confirmed for a variety of more general Ramsey models. ${ }^{1}$ Another natural source of distortions is private information. The resulting incentive problems generate permanent intertemporal distortions, as the recent work on dynamic contracting has emphasized. ${ }^{2}$ For other frictions, such as selfenforcement, limited commitment, political economy, incomplete markets and so on, results differ on the desirability of long-run intertemporal distortions.

Why are permanent intertemporal distortions ruled out in some environments? What is different about economies where they are optimal? To explore these questions for a broad class of second best economies, we propose a unified framework that encompasses and extends many well known models. We allow for a general formulation of the constraints on resource allocation, which we refer to as admissibility constraints. We can capture variants of the Ramsey taxation model with aggregate or idiosyncratic risk, as well as economies with incentive compatibility constraints due to limited commitment, political economy, self-enforcement or private information. Our framework includes also settings that combine these constraints with arbitrary restrictions, such as incomplete markets and borrowing constraints on the government or on private agents.

Our general analysis uncovers a common optimality principle linking the intertemporal allocation of resources with the ability to front-load distortions. Specifically, the only rationale for intertemporal distortions is to relax future admissibility constraints. We show that when it is possible to front-load all distortions, future admissibility constraints eventually will stop binding and intertemporal distortions will be temporary. Front-loading all distortions may not be possible in some second best economies, and intertemporal distortions may then be permanent.

Formally, we identify a sufficient condition that rules out permanent intertemporal distortions: If there exists an allocation that satisfies all admissibility constraints and that eventually converges to the limiting first best allocation, then intertemporal distortions are temporary in the second

\footnotetext{
${ }^{1}$ Chari and Kehoe (1999) and Atkeson, Chari and Kehoe (1999) extend the Chamley-Judd result for a broad class of deterministic economies. Zhu (1992) and Farhi (2007) show that it holds also with aggregate uncertainty for complete and incomplete markets, respectively.

${ }^{2}$ Diamond and Mirrlees (1978) and Rogerson (1985) were the first to show that intertemporal distortions arise in dynamic disability insurance and moral hazard models. Golosov, Kocherlakota and Tsyvinski (2003) prove that intertemporal distortions prevail in a large class of private information economies.
} 
best. Importantly, the sufficient condition is a property of the set of admissible allocations, and not a property of the optimum. The condition does not imply that the second best will converge to the first best limiting allocation. Intratemporal distortions depend on the history of past binding constraints and typically do not vanish in the long run.

For example, our sufficient condition is satisfied in the standard Ramsey taxation model. Since the government can save enough to finance all its expenditures from asset returns, eventually it becomes possible to eliminate distortionary taxes and converge to the first best steady state. Intertemporal distortions are temporary, but typically intratemporal distortions, such as labor income taxes, will be present in the steady state. By contrast, the sufficient condition generally does not hold in private information economies, since the incentive compatibility constraints are not satisfied at the first best allocation. The need to relax future binding incentive compatibility constraints generates a rationale for distorting the intertemporal margin. This leads to permanent intertemporal distortions and a front-loaded path of consumption.

Of course, since our condition is not necessary, there are economies with no intertemporal distortions where the condition does not hold. The Ramsey model with a balanced budget is one example. The sufficient condition is not satisfied, yet capital income taxes may be zero in the long run and in this case, intertemporal distortions will be temporary. ${ }^{3}$

This paper makes several contributions. Our unified framework offers insight about how the nature of admissibility constraints shapes intertemporal distortions. This can prove useful for both normative and positive analysis of second best allocations. Consider intertemporal distortions from a normative standpoint. The Ramsey framework has been challenged for its arbitrary restrictions on fiscal instruments. Deriving constraints from primitive frictions, such as private information, ensures all relevant trade-offs are considered and instills greater confidence in the resulting policy prescriptions. ${ }^{4}$ Yet, this approach does not identify the relevant trade-off missing in the Ramsey model. Our result makes clear that the ability to front-load all distortions in the Ramsey model gives rise to this fundamental difference, even as the optimal allocation front-loads distortions in both environments. This finding fosters a deeper understanding of both models. In addition, our focus on distortions rather than taxes leads to more general predictions. ${ }^{5}$

From a positive standpoint, our sufficient condition is useful for establishing robust predictions on the long-run properties of the intertemporal margin for different types of frictions. For example, for economies with self-enforcement constraints, we show how the assumptions on the agents' outside option determine whether the sufficient condition holds, and thus set the long run properties of intertemporal distortions. This analysis reconciles the conflicting findings in the literature. We are able also to derive a very general result for economies with a benevolent government who chooses allocations under limited commitment. Our sufficient condition holds in this environment, as the

\footnotetext{
${ }^{3}$ See Judd (1985) and Lansing (1999).

${ }^{4}$ See Kocherlakota (2005a) for an extensive discussion on this point.

${ }^{5}$ It also clarifies some contrasting results in the literature that are driven by specific assumptions on the decentralization. As is well known, there may be many ways to implement constrained-efficient allocations with a combination of taxes and trading restrictions in a decentralized arrangement. See Kocherlakota (2005b), Albanesi and Sleet (2006), and Albanesi (2008) for a discussion.
} 
first best allocation must dominate the outside option. Thus, limited commitment alone cannot give rise to permanent intertemporal distortions.

Finally, the sufficient condition is easy to verify and can be employed immediately to determine whether a specific economy features permanent intertemporal distortions, without having to fully characterize the solution. This is a big advantage, since many interesting second best environments are rich and present considerable analytical and computational challenges. Knowledge of the limiting behavior of the intertemporal margin can also be helpful when we want to solve the model fully, since this property of the solution can be incorporated into the algorithm.

The paper is organized as follows. To illustrate possible admissibility constraints, Section 2 presents a number of examples of well known second best economies. Section 3 lays out the general framework. The economy is populated by infinitely lived agents with standard time separable preferences defined over consumption and labor. We allow for ex ante and ex post heterogeneity. The production function is neoclassical and exhibits constant returns to scale, implying that the first best allocation is fully efficient and does not entail any intrinsic distortions. The second best problem is formulated as a choice of feasible allocations subject to admissibility constraints. ${ }^{6}$ The admissibility constraints are forward-looking and display limited history dependence. We also assume a number of regularity conditions. These assumptions correspond to a generalized version of those typically imposed in the particular second best economies that we aim to encompass. Specifically, we consider exclusively economies where the second best allocation is interior and weakly converges. Since the set of admissible allocations often is not convex, we generalize a condition in Ray (2002) that rules out local maxima and inflexion points.

Section 4 formally proves the main result. The argument can be outlined as follows. We decompose the second best problem into two stages. The first stage shows that if all future admissibility constraints on the allocation stop binding, the optimal allocation will feature only temporary intertemporal distortions. The proof of this result is closely related to Zhu (1992). The sufficient condition plays a role in the second stage. We show that if it holds, the second best allocation is such that all future admissibility constraints eventually stop binding. Our result follows.

Section 5 discusses several applications that show the significance and applicability of our result. Section 6 concludes.

\section{Examples}

We now present a number of canonical examples of second best economies designed to illustrate the structure of the different admissibility constraints. We also include a short discussion noting whether our sufficient condition is satisfied in these models.

\footnotetext{
${ }^{6}$ This formulation follows in the tradition of the primal approach to optimal taxation, pioneered in Atkinson and Stiglitz (1980) and Lucas and Stokey (1983).
} 


\subsection{Ramsey taxation model}

Taxes are perhaps the most immediate source of distortions in the allocation of resources. The Ramsey model has been the benchmark for the analysis of optimal taxation in macroeconomics. ${ }^{7}$ In this framework, a benevolent government sets proportional labor and capital income taxes to finance an exogenous stream of government consumption. The government has commitment and can issue debt of different maturities. Crucially, lump-sum taxes are ruled out, which implies that the first best allocation cannot be attained.

The constraints on fiscal instruments that define the Ramsey approach generally can be expressed in terms of allocations, an approach that greatly simplifies the analysis. For example, admissible allocations in a Ramsey model with a representative agent and no uncertainty are fully characterized by the following implementability constraint:

$$
\sum_{t=0}^{\infty} \beta^{t}\left(u_{t}^{c} c_{t}+u_{t}^{l} l_{t}\right) \geq u_{0}^{c}\left\{\left[\left(1-\tau_{0}^{k}\right) r_{0}+1-\delta\right] k_{0}+b_{0}\right\} .
$$

This constraint is derived by combining the government intertemporal budget constraint with the competitive equilibrium conditions for households and firms. Since consumption and labor decisions in the future affect the present value of tax revenues, the implementability constraint is forwardlooking.

In stochastic economies, admissible allocations can be characterized also in terms of a single implementability constraint as long as markets are complete. However, if the government can issue only risk-free debt, it faces a sequence of implementability constraints, one for each possible history of shocks:

$$
E_{t}\left\{\left(u_{t}^{c}\right)^{-1} \sum_{d=t}^{\infty} \beta^{d-t}\left(u_{d}^{c} c_{d}+u_{d}^{l} l_{d}\right)-R_{t} k_{t}\right\}=b_{t-1}+V_{t},
$$

where $b_{t-1}$ is the return on debt carried into period $t, V_{t}$ is the present value of non-negative transfers, and $R_{t}$ is the after-tax return on capital. ${ }^{8}$ Even if constraint (2) cannot be expressed solely in terms of allocations, the dynamics of debt are decoupled from the forward-looking component that involves the continuation allocation.

Ramsey models generally satisfy the sufficient condition. As long as the government can save, there exists an allocation that converges to the first-best steady state. More precisely, it is possible to design a policy in which tax revenues exceed government spending until the income from government assets grows enough to finance government expenditures. At this point, there is no further need to raise tax revenues, and since taxes are the only source of distortions, the steady state that would prevail under lump sum taxes can be attained. We discuss this property more extensively in Section 5.1 .

\footnotetext{
${ }^{7}$ Chari and Kehoe (1999) provide an excellent review of this approach.

${ }^{8}$ See Aiyagari, Marcet, Sargent and Seppala (2003) and Farhi (2007) for an analysis of the Ramsey model under incomplete markets. For simplicity we have omitted the dependence on each variable on the history of shocks.
} 


\subsection{Risk Sharing}

Incentive-constrained risk sharing is a classic example of a second best problem. Ex ante identical agents face idiosyncratic shocks to productivity or preferences. The first best allocation provides agents with full insurance against these shocks. However, additional frictions such as limited commitment or asymmetric information imply that full insurance typically is not attainable.

\subsubsection{Self-enforcement constraints}

Kocherlakota (1996) is perhaps one of the best known models of incomplete risk sharing under limited commitment. To demonstrate the implications of this friction, we extend his environment to include capital accumulation.

The economy is populated by two ex ante identical agents, $i=1,2$, infinitely lived, with standard preferences defined over sequences of consumption, $c$, and labor, $l$. The agents face idiosyncratic risk in their labor productivity, $\theta$. Let $\theta_{t} \in\{\underline{\theta}, \bar{\theta}\}$ with $\underline{\theta}<\bar{\theta}$ and $\operatorname{Pr}(\underline{\theta})=\operatorname{Pr}(\bar{\theta})=0.5$. The social welfare function is

$$
E_{t}\left\{\sum_{i=1,2} \gamma_{i} \sum_{t=0}^{\infty} \beta^{t} u\left(c_{i, t}, l_{i, t} / \theta_{t}\right)\right\},
$$

where $\gamma_{i}$ denotes the Pareto weight on agent $i$. Resource feasibility requires:

$$
c_{1, t}+c_{2, t}+k_{t+1} \leq F\left(k_{t}, l_{t}\right)
$$

for all $t \geq 0$, where $l_{t}=l_{1, t}+l_{2, t}$ denotes aggregate labor in efficiency units. The production function $F(\cdot)$ is increasing in both arguments, strictly concave and homogeneous of degree one.

The first best allocation fully insures agents from their idiosyncratic shocks. The ratio of the marginal utility of consumption across agents is constant over time, and for each agent the marginal rate of substitution between consumption and labor is equal to their labor productivity. Thus, high productivity agents supply more labor for standard preferences.

Under limited commitment, an agent may have an incentive to deviate from a risk-sharing arrangement in a state when she has high productivity. If she deviates, she has access to an outside option, $V_{\text {out }}\left(k_{t}\right)$, which we assume to depend only on the level of aggregate capital. ${ }^{9}$ Thus any second best allocation must satisfy a self-enforcement constraint that imposes for each date that the present value of utility from continuing with the allocation is greater that the utility associated with the outside option. Formally:

$$
E_{t}\left\{\sum_{j=0}^{\infty} \beta^{j} u\left(c_{t+j}^{i}, l_{t+j}^{i} / \theta_{t+j}\right)\right\} \geq V_{\text {out }}\left(k_{t}\right),
$$

for $i=1,2$ and $\forall t \geq 0$.

\footnotetext{
${ }^{9}$ There are many possible formulations of the outside option, which we discuss in detail in Section 5.2.1.
} 
The self-enforcement constraint restricts the continuation allocation in each period, and thus is forward looking. Each agent's self-enforcement constraint depends only on the value of the outside option and her own continuation allocation. The constraint can be relaxed by allocating more consumption in the future.

Let $\left\{c_{i, t+j}^{f b}, l_{i, t+j}^{f b}\right\}_{j \geq 0}\left(k_{t}\right)$ be a resource-feasible allocation that maximizes social welfare from time $t$ onwards with aggregate capital given by $k_{t}$. We will refer to this object as a continuation first best allocation from $k_{t}$. The sufficient condition holds for this model if the first-best continuation allocation satisfies the self-enforcement constraint, that is:

$$
E_{t}\left\{\sum_{j=0}^{\infty} \beta^{j} u\left(c_{i, t+j}^{f b}, l_{i, t+j}^{f b} / \theta_{t+j}\right)\right\} \geq V_{\text {out }}\left(k_{t}\right)
$$

for $i=1,2$.

Since the continuation first best allocation features full consumption insurance, at the utilitarian first-best allocation, the sufficient condition is satisfied for high enough values of $\beta$, as in Kocherlakota (1996). If however Pareto weights are sufficiently asymmetric then condition (4) may not be satisfied even if $\beta$ approaches 1.

\subsubsection{Private information}

We now consider the same economy under the assumption that the idiosyncratic preference shocks, $\theta$, are private information. This is a version of the environment considered in Golosov, Kocherlakota and Tsyvinski (2003), without aggregate shocks. The first best allocation is the same as in the previous example.

Agents at time $t$ are characterized by their history of productivity shocks, denoted with $\theta^{t}=$ $\left\{\theta_{0}, \theta_{1}, \ldots, \theta_{t}\right\}$. Thus, an allocation is a mapping that specifies consumption as a function of the history: $\left\{c_{i}\left(\theta^{t}\right), l_{i}\left(\theta^{t}\right)\right\}_{t \geq 0}^{i=1,2}$. Private information implies that allocations must satisfy a truth-telling requirement known as incentive compatibility constraint:

$$
E_{t}\left\{\sum_{t=0}^{\infty} \beta^{t} u\left(c_{i}\left(\theta^{t}\right), l_{i}\left(\theta^{t}\right) / \theta_{t}\right)\right\} \geq E_{t}\left\{\sum_{t=0}^{\infty} \beta^{t} u\left(c_{i}\left(\tilde{\theta}^{t}\right), l_{i}\left(\tilde{\theta}^{t}\right) / \theta_{t}\right)\right\}
$$

$\forall \tilde{\theta}^{t}, \theta^{t}$ and for $i=1,2$. This constraint is forward looking and involves restrictions across allocations awarded to different ex post types, $\theta^{t}$. Capital does not enter this constraint directly, in contrast to (3).

Incentive compatibility requires individual consumption to be correlated with the realized endowment. Absent this correlation, agents will report a low value of $\theta$. The first best allocation does not satisfy (5) if utility is strictly concave and the endowment process does not exhibit any absorbing states. Thus, the sufficient condition typically does not hold. 


\section{General Framework}

We start by describing the economy and defining the best feasible allocation, which corresponds to our notion of first best. We then introduce our general formulation for the admissibility constraints and the corresponding concept of second best allocation.

\subsection{The Economy}

We consider an infinite-horizon economy where time is discrete $t=0,1, \ldots$ The economy is populated by a continuum of agents who may be ex-ante heterogeneous. Let $I$ be the finite set of ex-ante types, each denoted by subscript $i \in I$, and $\pi_{i}>0$ the measure of agents of ex-ante type $i$, with $\sum_{I} \pi_{i}=1$.

Aggregate shocks are denoted by $z_{t} \in Z$, where $Z$ is a finite set. Let $z^{t}=\left\{z_{0}, z_{1}, \ldots, z_{t}\right\}$ be the history of aggregate shocks from date 0 up to date $t$, and $z^{\infty} \in Z^{\infty}$ denotes an infinite history. The set of continuation histories from node $\tilde{z}^{d}$ at a later date $t \geq d$ is denoted with:

$$
Z^{t} \mid \tilde{z}^{d} \equiv\left\{z^{t} \in Z^{t} \mid z^{d}=\tilde{z}^{d}\right\}
$$

We adopt this notational convention for all variables.

Aggregate shocks are governed by a first-order Markov process $\pi_{z}$. The probability measure of a node $z^{t}$ given past history $\tilde{z}^{d}$ is denoted $\pi_{z}\left(z^{t} \mid \tilde{z}_{d}\right)$. Since $Z$ is a finite set the probability measure is well defined for all subsets of $Z^{t}$ and $Z^{\infty}$.

We also allow for idiosyncratic shocks, so agents may be ex-post heterogeneous. Let $\theta_{t} \in \Theta$ denote the idiosyncratic shock at date $t$, where $\Theta$ is a finite set. The agent type at any date $t$ is given by the ex-ante type $i$ and the history of idiosyncratic shocks $\theta^{t} \in \Theta^{t}$.

Idiosyncratic shocks are governed by the first-order Markov process $\pi_{\theta}$ and are independent from the aggregate shock $z_{t}$ and the agent ex-ante type. We also assume a law of large numbers holds, so $\pi_{\theta}\left(\theta^{t}\right)$ is also the measure of agents with type $\theta^{t}$ at date $t$.

Let $s_{t}=\left\{z_{t}, \theta_{t}\right\}$, with $s_{t} \in S=Z \times \Theta$, summarize the aggregate state and idiosyncratic state. Since aggregate and idiosyncratic shocks are independent, state $s_{t}$ evolves stochastically according to a first-order Markov process with probability distribution $\pi\left(s^{t}\right)=\pi_{z}\left(z^{t}\right) \pi_{\theta}\left(\theta^{t}\right)$.

We assume that the Markov transition matrices for both aggregate and idiosyncratic shocks are strictly positive component-wise. This guarantees that there is a unique ergodic distribution associated with the exogenous state $s_{t} .{ }^{10}$

Assumption 1 For all $s, s^{\prime} \in S, \pi\left(s^{\prime} \mid s\right)>0$.

Let $c_{i}\left(z^{t}, \theta^{t}\right)$ be the consumption of ex-ante type $i \in I$ after a sequence of idiosyncratic shocks $\theta^{t} \in \Theta^{t}$ and aggregate shocks $z^{t} \in Z^{t}$. To economize on notation, we will write $c_{i}\left(s^{t}\right)$ whenever

\footnotetext{
${ }^{10}$ See Theorem 11.4 in Stokey and Lucas (1989). Our results also hold for Markov processes with absorbing states and multiple ergodic distributions. We adopt this more restrictive formulation for ease of exposition.
} 
there is no confusion possible. Consumption is non-negative and bounded above by $\bar{c}>0$ at all dates,

$$
c_{i}\left(z^{t}, \theta^{t}\right) \in[0, \bar{c}]
$$

Let $C \equiv[0, \bar{c}]^{|I \times \Theta|}$ where $|I \times \Theta|$ denotes the cardinality of the set. The distribution of consumption across ex-ante types at a given node $s^{t}$ is denoted with:

$$
c\left(s^{t}\right)=\left\{c_{i}\left(s^{t}\right) \in[0, \bar{c}]: i \in I\right\} .
$$

A consumption allocation, $c$, is a plan specifying consumption for every ex-ante type at every node $s^{t}:$

$$
c=\left\{c\left(s^{t}\right): s^{t} \in S^{t}, t \geq 0\right\}
$$

Hence, $c \in C^{\infty}$ and since $C$ is compact, $c \in l_{\infty}$ with the sup norm.

A continuation consumption allocation from node $s^{t}$ or $s^{t} \in S^{t}$ and $t>0$, denoted with $c \mid s^{t}$, is a plan specifying consumption for every ex ante type at each continuation node from $s^{t}$ :

$$
c \mid s^{t}=\left\{c\left(s^{d}\right): s^{d} \in S^{d} \mid s^{t}, d \geq t\right\} .
$$

Labor in efficiency units is denoted $l_{i}\left(z^{t}, \theta^{t}\right) \in[0, \bar{l}]$. Following the notational convention for consumption, similarly we can define $l\left(s^{t}\right), L, l$, and $l \mid s^{t}$. It will be useful to collect the consumption and labor allocation in one variable, which we will refer to as flow allocation:

$$
x_{i}\left(z^{t}, \theta^{t}\right)=\left\{c_{i}\left(z^{t}, \theta^{t}\right), l_{i}\left(z^{t}, \theta^{t}\right)\right\}
$$

We let $X=C \times L$ and follow the notational convention to define $x\left(s^{t}\right), x$, and $x \mid s^{t}$.

Let $k\left(z^{t-1}\right) \in[0, \bar{k}] \equiv K$ denote the amount of aggregate capital available at node $z^{t} \in Z^{t} \mid z^{t-1}$. An allocation for aggregate capital is then given by:

$$
k=\left\{k\left(z^{t}\right): z^{t} \in Z^{t}, t \geq 0\right\},
$$

for given initial value, $k_{-1}$. The allocation for aggregate capital, $k$, belongs to $K^{\infty} \in l_{\infty}$. A continuation allocation for aggregate capital will be denoted with $k \mid z^{t}$.

The allocation for individual capital is denoted with $y$. Specifically, let $y_{i}\left(s^{t-1}\right) \in[0, \bar{y}]$ be the amount of capital held by ex-ante type $i$ at node $s^{t-1}$, and

$$
y\left(s^{t-1}\right)=\left\{y_{i}\left(s^{t-1}\right) \in[0, \bar{y}]: i \in I\right\},
$$

be the distribution of capital across ex-ante agents at node $s^{t-1}$. The initial distribution of individual capital, $y_{-1}$, is taken as given. The allocation of individual capital is:

$$
y=\left\{y\left(z^{t}\right): z^{t} \in Z^{t}, t \geq 0\right\}
$$


Definition 1 An allocation $\psi$ is a triple $\{x, y, k\} \in X^{\infty} \times Y^{\infty} \times K^{\infty}$. Let $\Psi$ be the set of all such allocations.

Given an allocation $\psi$, we can construct a corresponding sequence of probability measures $\left\{\mu_{t}\right\}_{t=0}^{\infty}$ over the Borel sets of $X^{\infty} \times Y^{\infty} \times K^{\infty}$. We will use the notation $\mu_{t} \rightarrow \mu_{\infty}$ to indicate that the sequence $\left\{\mu_{t}\right\}_{t=0}^{\infty}$ converges weakly to the probability measure $\mu_{\infty}$, and we will refer to $\mu_{\infty}$ as the limiting measure. ${ }^{11}$

Output is produced by combining labor and capital according to a constant-returns-to-scale production function $F$. The resource constraint at node $z^{t}$ is

$$
\mathbf{c}\left(z^{t}\right)+k\left(z^{t}\right)+g\left(z_{t}\right) \leq F\left(k\left(z^{t-1}\right), \mathbf{l}\left(z^{t}\right) ; z_{t}\right)
$$

where

$$
\begin{aligned}
\mathbf{c}\left(z^{t}\right) & =\sum_{i \in I} \sum_{\theta^{t} \in \Theta^{t}} \pi_{i} \pi_{\theta}\left(\theta^{t}\right) c_{i}\left(s^{t}\right), \\
\mathbf{l}\left(z^{t}\right) & =\sum_{i \in I} \sum_{\theta^{t} \in \Theta^{t}} \pi_{i} \pi_{\theta}\left(\theta^{t}\right) l_{i}\left(s^{t}\right),
\end{aligned}
$$

are aggregate consumption and labor respectively, and $g: Z \rightarrow[0, \bar{g}]$ is an exogenous process for government expenditures. The production function includes capital depreciation and satisfies standard properties, as detailed in Assumption 2.

Assumption 2 The production function $F$ is homogeneous of degree one, twice differentiable, concave and strictly increasing in labor and capital.

Feasibility also requires that the distribution of capital across agents be consistent with aggregate capital:

$$
\sum_{i \in I} \pi_{i}\left\{\sum_{\theta^{t} \in \Theta^{t}} \pi_{\theta}\left(\theta^{t}\right) y_{i}\left(s^{t}\right)\right\} \leq k\left(z^{t}\right) .
$$

Constraint (7) can be slack, to allow for a fraction of aggregate capital to be unassigned to any individual agent.

Definition 2 An allocation $\psi \in \Psi$ is feasible if the resource constraint (6) and the capital allocation constraint (7) are satisfied for all $z^{t} \in Z^{t}, t \geq 0$. Let $\Psi^{F} \subset \Psi$ be the set of feasible allocations.

For continuation allocations, feasibility must be defined from a particular state $\left\{s^{t}, k\left(z^{t-1}\right)\right\}$. A continuation allocation $\psi \mid s^{t}$ is feasible from state $\left\{s^{t}, k\left(z^{t-1}\right)\right\}$, if the resource constraint (6) and the capital allocation constraint (7) are satisfied for all $z^{d} \in Z^{d} \mid z^{t}, d \geq t$, for given $k\left(z^{t-1}\right) \in K$.

\footnotetext{
${ }^{11}$ A sequence $\left\{\mu_{t}\right\}_{t=0}^{\infty}$ converges weakly to a probability measure $\mu_{\infty}$ if $\lim _{t \rightarrow \infty} \mu_{t}(B)=\mu_{\infty}(B)$ for all Borel sets $B$ of $K \times X \times Y$ with $\mu_{\infty}(\partial B)=0$. Equivalently, $\lim _{t \rightarrow \infty} \int f d \mu_{t}=\int f d \mu_{\infty}$ for all continuous, bounded functions $f: K \times X \times Y \rightarrow \Re$.
} 
Individual preferences over flow allocations are represented by :

$$
U_{i}\left(x ; z_{0}\right)=\sum_{t=0}^{\infty} \sum_{s^{t} \in S^{t}} \beta^{t} \pi\left(s^{t}\right) u_{i}\left(x_{i}\left(s^{t}\right) ; \theta_{t}\right)
$$

where $\beta \in(0,1)$ is the intertemporal discount factor and per-period utility function $u_{i}$ satisfies the standard properties. While this formulation allows for heterogeneity in preferences, all agents are restricted to share the same discount factor.

Assumption 3 For all $i \in I$ the utility function $u_{i}:[0, \bar{c}] \times[0, \bar{l}] \times \Theta \rightarrow \Re$ is concave, twice differentiable, strictly increasing in consumption, and decreasing in labor.

The social welfare function is represented by:

$$
U\left(x ; z_{0}\right)=\sum_{i \in I} \pi_{i} U_{i}\left(x ; z_{0}\right)
$$

with $U: X^{\infty} \rightarrow \Re$. This formulation can accommodate arbitrary Pareto weights over ex-ante types $I$, which could be captured in the definition of $U_{i}(\cdot)$.

We can now provide a formal definition of a first best allocation.

Definition 3 A feasible allocation $\psi^{f b} \in \Psi^{F}$ is first best given initial conditions $k_{0}$ and $z_{0}$, if for all feasible allocations $\psi \in \Psi^{F}$ :

$$
U\left(x^{f b} ; z_{0}\right) \geq U\left(x ; z_{0}\right)
$$

A continuation first best allocation from state $\left\{s^{t}, k\left(z^{t-1}\right)\right\}$ for $s^{t} \in S^{t}$ and $k\left(z^{t-1}\right) \in K$, denoted with $\psi^{f b} \mid\left\{s^{t}, k\left(z^{t-1}\right\}\right.$, is a feasible continuation allocation from $\left\{s^{t}, k\left(z^{t-1}\right)\right\}$ that satisfies:

$$
U\left(x^{f b} \mid\left\{s^{t}, k\left(z^{t-1}\right)\right\}, z_{t}\right) \geq U\left(x \mid\left\{s^{t}, k\left(z^{t-1}\right)\right\}, z_{t}\right)
$$

for all feasible continuation allocations $x \mid\left\{s^{t}, k\left(z^{t-1}\right)\right\}$.

The distribution of capital across agents is indeterminate in the first best, since it is inconsequential for welfare or production efficiency under our assumptions. We can thus define the sequence of probability measures associated with the first best allocation, $\left\{\mu_{t}^{f b}\right\}$, and its limit $\mu_{\infty}^{f b}$ over $X \times K$, with the understanding that for any first best allocation, $\psi^{f b}$, the associated sequence of probability measures over $X \times K$ converges weakly to $\mu_{\infty}^{f b}$. This also implies that for any first best continuation allocation, $\psi^{f b} \mid s^{t}$, the associated sequence of probability measures converges to $\mu_{\infty}^{f b}$.

\subsection{Admissibility constraints}

For the first best allocation, feasibility is the only constraint on the allocation of resources. Most interesting economic environments feature additional constraints, as discussed in Section 2, which we refer to as admissibility constraints. We now introduce a general and abstract formulation for 
the admissibility constraints that captures a large class of second best economies. Our general formulation comprises two classes of constraints. There are constraints on the allocation, possibly at each date and state and for each ex ante type of agent. These are parameterized by an auxiliary variable, which does not enter preferences or production but which may be necessary to formulate the second best problem. The auxiliary variable is endogenous and may be subject to initial conditions and to constraints on its law of motion. These make up the second set of constraints covered by our general formulation.

Specifically, at each date $t \geq 0$, for each aggregate shock history $z^{t} \in Z^{t}$, each ex-post type $\theta^{t} \in \Theta^{t}$, and each ex-ante type $i \in I$, there are $\{1,2, \ldots, M\}$ admissibility constraints on the allocation, where $M$ is a finite number. We represent constraint $\{m, i\}$ at node $s^{t}$ with:

$$
H_{m, i}\left(\psi, s^{t}\right) \leq a_{m, i}\left(s^{t}\right),
$$

where $a_{m, i}\left(s^{t}\right)$ is the value of the auxiliary variable, $a$, that parameterizes the constraint $m, i$ at node $s^{t}$.

The function $H_{m, i}\left(\psi, s^{t}\right)$ is given by:

$$
H_{m, i}\left(\psi, s^{t}\right)=\mathbf{b}_{m, i}\left(\psi, s^{t}\right)+\mathbf{h}_{m, i}^{0}\left(\psi, s^{t}\right)+\sum_{\tilde{s}^{t} \in S^{t} \mid s^{t-1}} \mathbf{h}_{m, i}^{1}\left(\psi, \tilde{s}^{t}\right)
$$

where

$$
\begin{aligned}
& \mathbf{b}_{m, i}\left(\psi, s^{t}\right)=b_{m, i}\left(x\left(s^{t}\right), y\left(s^{t-1}\right) ; k\left(z^{t-1}\right), s_{t}\right) \\
& \mathbf{h}_{m, i}^{0}\left(\psi, s^{t}\right)=d_{m, i}^{0}\left(x\left(s^{t}\right) ; s_{t}\right) \sum_{j=t+1}^{\infty} \sum_{s^{j} \in S^{j} \mid s^{t}} \beta^{j-t} \pi\left(s^{j} \mid s_{t}\right) h_{m, i}^{0}\left(x_{i}\left(s^{j}\right) ; s_{j}\right), \\
& \mathbf{h}_{m, i}^{1}\left(\psi, \tilde{s}^{t}\right)=d_{m, i}^{1}\left(x\left(s^{t}\right) ; s_{t}\right) \sum_{j=t+1}^{\infty} \sum_{s^{j} \in S^{j} \mid \tilde{s}^{t}} \beta^{j-t} \pi\left(s^{j} \mid \tilde{s}_{t}\right) h_{m, i}^{1}\left(x_{i}\left(s^{j}\right) ; s_{j}\right),
\end{aligned}
$$

with

$$
\begin{aligned}
b_{m, i} & : \quad[0, \bar{x}]^{I} \times[0, \bar{y}]^{I} \times K \times S \rightarrow \Re, \\
h_{m, i}^{j} & : \quad[0, \bar{x}] \times S \rightarrow \Re, \\
d_{m, i}^{j} & : \quad[0, \bar{x}] \times S \rightarrow \Re,
\end{aligned}
$$

for $j \in\{0,1\}$. For convenience, we stack each value of $H_{m, i}\left(\psi ; s^{t}\right)$ into $H_{i}\left(\psi ; s^{t}\right)$ and then into $H\left(\psi ; s^{t}\right)$. We assume that each component function of $H$ is twice differentiable.

Assumption 4 Functions $\left\{b_{m, i}, h_{m, i}^{j}, d_{m, i}^{j}\right\}$ are twice differentiable in every argument for all $i \in I$, $m \in M$.

The vector:

$$
a\left(s^{t}\right)=\left\{a_{m, i}\left(s^{t}\right) \in[0, \bar{a}]: i \in I, m \in M\right\}
$$


collects the relevant values of the auxiliary variable for node $s^{t} \in S^{t}, t \geq 0$. Let $A=[0, \bar{a}]^{|I \times M|} \subset$ $\Re^{|I \times M|}$ be the space for $a\left(s^{t}\right) \in A$. A plan for the auxiliary variable is given by:

$$
a=\left\{a\left(s^{t}\right): s^{t} \in S^{t}, t \geq 0\right\} \in A^{\infty} .
$$

The auxiliary variable corresponds to assets or other endogenous state variables that do not enter production or preferences but are necessary for defining the second best allocation problem. The implementability constraints in the Ramsey model with incomplete markets in equation (2) are one example of this use. In this case, the auxiliary variable can be defined as a vector comprising values of $b_{t-1}$ and $V_{t}$. It can also capture time, state and agent variation in the admissibility constraints (9). The path of the auxiliary variable may itself be subject to constraints, stemming from asset trading restrictions and so on. We formalize these as follows. We say that a plan for the auxiliary variable is admissible if for all $z^{t} \in Z^{t}, \theta^{t} \in \Theta^{t}$,

$$
a\left(s^{t}\right) \in \Gamma\left(a\left(s^{t-1}\right) ; s_{t}\right)
$$

with $a_{0} \in A_{0} \subseteq A$ at date $t=0$.

The correspondence $\Gamma: A \times S \rightrightarrows A$ governs the law of motion for the auxiliary variable. We restrict $\Gamma$ to be convex. Since for most second best economies, the auxiliary variable will correspond to assets with linear laws of motion, the condition will be satisfied for most problems of interest.

Assumption 5 The correspondence $\Gamma(\alpha, s): A \times S \rightrightarrows A$ is continuous, convex, and its image is a convex subset of $\Re^{I \times M}$ including $\alpha$ for all $s \in S$ and $\alpha \in A$.

We can now formally define the notion of a second best allocation for our economy.

Definition $4 A$ feasible allocation $\psi \in \Psi^{F}$ is admissible if there exists a plan for the auxiliary variable $a \in A$, with $a_{0} \in A_{0}$, such that for all dates $t \geq 0$, all $s^{t} \in S^{t}$ : (i) the allocation, $\psi$, satisfies (9) for $m=1,2, \ldots, M$ and $i=1,2, \ldots, I$; (ii) the plan for the auxiliary variable, a, satisfies (11). Let $\Psi^{A}$ be the set of admissible allocations.

Definition 5 A second best allocation is an admissible allocation $\psi^{*}$ given initial conditions $k_{0}$ and $z_{0}$, if for all admissible allocations $\psi \in \Psi^{A}$ :

$$
U\left(x^{*} ; z_{0}\right) \geq U\left(x ; z_{0}\right)
$$

If the first best allocation $\psi^{f b}$ is admissible, the admissibility constraints will never be binding and the second best problem is trivial. To distinguish this case, we introduce the concept of proper second best. This corresponds to a second best allocation that satisfied: $U\left(x^{*} ; z^{0}\right)<U\left(x^{f b} ; z^{0}\right)$.

Let us briefly discuss our formulation for the admissibility constraints (9) and (11). The constraints on the allocation (9) comprise three terms. The term $\mathbf{b}_{m, i}$ depends on the state of the 
economy $\left\{k\left(z^{t-1}\right), s_{t}\right\}$, and on the agent's decisions and type. For example, the term $\mathbf{b}_{m, i}$ can capture the value of an outside option for economies with limited commitment constraints on the government or private agents. The term $\mathbf{h}_{m, i}^{0}$ is purely forward-looking, since it includes only the continuation allocation for consumption and labor. This term is intended to capture admissibility constraints that have this feature, such as the implementability constraints in Ramsey taxation models or the value of an allocation on the equilibrium path for a setting with participation conditions. Finally, the term in $\mathbf{h}_{m, i}^{1}$ allows us to incorporate constraints on the allocation awarded to different ex post types of agents. Such restrictions arise in settings with constraints on redistribution or risk-sharing. Implementability constraints in private information economies are a typical example, as discussed in Section 2.2.2. ${ }^{12}$ The terms $d_{m, i}^{j}$ allow us to capture endogenous discount factors that depend on marginal utilities. These terms often appear in intertemporal budget constraints. Finally, the auxiliary variable enters linearly in the admissibility constraints (9). This is without loss of generality, as the auxiliary variable could be defined as dependent on endogenous variables. For example, it could be given by the product of asset holdings weighed by marginal utility, and this definition could itself be captured in (9). ${ }^{13}$

While the formulation of $H\left(\psi, s^{t}\right)$ enables us to capture a broad class of second best economies, it imposes structure on the degree of history dependence of the admissibility constraints. First, the constraint at each node cannot be arbitrarily backward-looking. Second, the value of endogenous state variables at future dates does not enter the admissibility constraint at the current date. These assumptions are clearly restrictive. For example, a Ramsey taxation model with the constraint that taxes must be constant over time cannot be captured in (9). ${ }^{14}$ Finally, we impose that the forward looking terms $\mathbf{h}_{m, i}^{0}$ and $\mathbf{h}_{m, i}^{1}$ are time-separable and discounted at the same rate as utility.

The specification for the constraints on the law of motion of the auxiliary variable, (11), is similarly motivated. The correspondence $\Gamma$ can capture constraints on individual or aggregate asset positions, or on their variation over time, as well as certain information constraints, such as measurability conditions. ${ }^{15}$ As before there are some limitations embedded in our formulation. The auxiliary variable must be multiplicatively separable with the terms in $H$, and the definition of $\Gamma$ clearly rules out arbitrary history dependent constraints.

To complete the description of our general framework, we introduce some additional conditions on the second best allocation. While in specific applications these assumptions can be stated in terms of primitives, it is very difficult to identify such primitive restrictions for our general framework.

The first assumption is a standard non-degenerate constraint qualification. This is required in

\footnotetext{
${ }^{12}$ The baseline formulation of $\mathbf{h}_{m, i}^{j}$ only allows for one period cross-agent restrictions on the allocation. However, it can easily be extended to include a finite number of terms $\mathbf{h}_{m, i}^{j}$.

${ }^{13}$ The implementability constraint in a Ramsey model is a typical example, see (1) and (2) in Section 2.1.

${ }^{14}$ The restrictions on the structure of (9) do not rule out Ramsey models with incomplete factor taxation, such as Correia (1996) and Jones, Manuelli and Rossi (1997). These restrictions can be formulated with an admissibility constraint as (9), by including an additional constraint at date $t=0$ that prevents the government from manipulating the present value of assets at date $t=0$. See Armenter (2007) for a discussion.

${ }^{15}$ The formulation of the correspondence $\Gamma$ can easily be extended to include a finite number lags of the auxiliary variable.
} 
order to apply Lagrangian methods and is based on the definition of regular point in Luenberger (1969). We assume also that the second best allocation is interior. This excludes constrainedefficient allocations that involve corner solutions. ${ }^{16}$

Assumption 6 Let $\psi^{*}$ be a second best allocation. Then for all nodes $s^{t} \in S^{t}, \psi^{*}$ is a regular point of $H_{m, i}\left(\psi, s^{t}\right)$ for all $i \in I, m \in M$, and belongs to the interior of $X \times K \times Y$.

The set of admissible allocations is not convex for many second best problems. The strategy for resolving this issue varies by application. Often, sufficiency is verified numerically or additional model-specific conditions are imposed to guarantee global optimality. ${ }^{17}$ Our formulation can encompass non-convex problems, under a regularity condition to rule out local maxima and inflexion points. We adopt a weaker and more general version of the conditions assumed in Ray (2002) for a dynamic principal-agent model with limited commitment. He imposes that any improvable admissible allocation can be improved upon locally. ${ }^{18}$ Since we focus on the limiting behavior of the allocations, we express the condition in terms of the limsup of the admissibility constraints. This formulation guarantees that any admissible allocation that can be improved upon globally can be eventually improved upon by a small amount. ${ }^{19}$

Assumption 7 Let $\psi^{f b}$ and $\psi^{*}$ be a first-best and second-best allocation respectively. For each $i \in I$ and $m \in M$ let

$$
\begin{aligned}
\kappa_{m, i}^{f b} & =\lim _{t \rightarrow \infty} \sup \left\{H_{m, i}\left(\psi^{f b}, s^{j}\right): s^{j} \in S^{j}, j \geq t\right\}, \\
\kappa_{m, i}^{*} & =\lim _{t \rightarrow \infty} \sup \left\{H_{m, i}\left(\psi^{*}, s^{j}\right): s^{j} \in S^{j}, j \geq t\right\} .
\end{aligned}
$$

Then:

$$
\kappa_{m, i}^{*} \leq \kappa_{m, i}^{f b}
$$

with strict equality only if $\mu_{\infty}^{*}=\mu_{\infty}^{f b}$.

Finally, we assume that the second best allocation converges weakly. As is well known, it is hard to identify sufficient conditions for convergence in many second best economies, and often it is necessary to impose additional restrictions on the problem to guarantee convergence. ${ }^{20}$ This difficulty is compounded in a general framework like ours. We follow most of the literature and assume that the second best allocation converges.

\footnotetext{
${ }^{16}$ For example, some private information economies display bunching. In this case, a subset of agents does not supply labor. See Tuomala (1990) and Salanie (2003) for a discussion. If there is heterogeneity in discount factors, consumption may converge to its lower bound in the second best. See Acemoglu, Golosov and Tsyvinski (2008a) for an example.

${ }^{17}$ For example, in private information models with labor choice, a single-crossing is imposed to ensure an ordering of the marginal rate of substitution between consumption and labor by types. This condition typically guarantees convexity.

${ }^{18}$ Assumption A2, page 556 in Ray (2002).

${ }^{19}$ In practice we can relax the assumption even further: we only need the regularity condition to be satisfied by binding admissibility constraints.

${ }^{20}$ For example, Judd (1985) presents the results conditional on convergence. Atkeson and Lucas (1995) introduce a lower bound on utility which guarantees convergence in an economy with moral hazard and private information.
} 
Assumption 8 Let $\left\{\mu_{t}^{*}\right\}$ be a sequence of probability measures associated with a second best allocation $\psi^{*}$. The sequence $\left\{\mu_{t}^{*}\right\}$ converges weakly to $\mu_{\infty}^{*}$.

\section{Main Result}

Our main result, derived in Theorem 1, identifies a sufficient condition that rules out permanent intertemporal distortions in the class of second best economies encompassed by our framework. The condition identifies a property of the set of the admissible allocations, thus it can be verified without solving for the second best allocation. It is also simple to state, given the generality of the environment.

Condition 1 There exists an admissible allocation $\tilde{\psi}$ such that the associated sequence of probability of measures $\left\{\tilde{\mu}_{t}\right\}$ converges weakly to $\mu_{\infty}^{\text {fb }}$ for any set of initial conditions $A_{0}$.

Condition 1 formalizes the idea that it is possible to front-load all distortions for some second best problems. Clearly, if there exists an admissible allocation that converges to the first best limiting allocation, for such an allocation all admissibility constraints must eventually be non-binding. The existence of such an allocation is a property of the set of admissible allocations and does not imply that this allocation solves the second best problem. However, Theorem 1shows that if Condition 1 holds, the second best allocation will not feature permanent intertemporal distortions.

We formalize the concept of intertemporal distortion as a wedge in the Euler equation for consumption. For a given an allocation $\psi$, let:

$$
\Delta_{i}\left(s^{t}\right)=u_{i}^{c}\left(s^{t}\right)-\beta \sum \pi\left(s^{t+1} \mid s_{t}\right) u_{i}^{c}\left(s^{t+1}\right) F^{k}\left(s^{t+1}\right),
$$

for all $i \in I$ and all $s^{t} \in S^{t}, t \geq 0$, where $u^{c}$ and $F^{k}$ denote the derivative of $u$ and $F$ with respect to $c$ and $k$ respectively. At the first best allocation, $\psi^{f b}, \Delta_{i}\left(s^{t}\right)=0$ for all agents and all nodes. For a second best plan $\psi^{*}$ we characterize only the limiting properties of the wedge $\Delta_{i}\left(s^{t}\right)$.

Abusing notation slightly, we extend the probability measure $\mu_{\infty}^{*}$ over events of the type $\Delta_{i}\left(s^{t}\right) \geq$ 0 as follows. Consider the function:

$$
f:[0, \bar{x}] \times[0, \bar{k}] \times S \times X^{\# Z} \rightarrow \Re,
$$

such that $f\left(x_{i}\left(s^{t}\right), k\left(s^{t}\right), \ldots\right)=\Delta_{i}\left(s^{t}\right)$. Let $\Upsilon_{i}$ the subset of the domain of $f$ such that $f \geq 0$. By Assumptions 2 and $3, f$ is continuous so $\Upsilon_{i}$ is measurable. We then use notation $\mu_{\infty}\left(\Delta_{i}\left(s^{t}\right) \geq 0\right)$ for $\mu_{\infty}\left(\Upsilon_{i}\right){ }^{21}$

We can now state our main result.

\footnotetext{
${ }^{21}$ Weak convergence $\mu_{t} \rightarrow \mu_{\infty}$ does not guarantee that $\lim _{t \rightarrow \infty} \mu_{t}\left(\Upsilon_{i}\right)=\mu_{\infty}\left(\Upsilon_{i}\right)$, so we formulate the proof in terms of $\left\{\Delta_{i}\left(s^{t}\right): s^{t} \in S^{t}, t \geq 0\right\}$.
} 
Theorem 1 Let $\psi^{*}$ be a second best allocation and $\mu^{*}$ be the associated sequence of probability measures. If Condition 1 holds, then for each $i \in I$ either the intertemporal distortion is zero on the limit with probability one,

$$
\mu_{\infty}^{*}\left(\Delta_{i}\left(s^{t}\right)=0\right)=1,
$$

or the intertemporal distortion fluctuates around zero,

$$
\begin{aligned}
& \mu_{\infty}^{*}\left(\Delta_{i}\left(s^{t}\right)>0\right)>0, \\
& \mu_{\infty}^{*}\left(\Delta_{i}\left(s^{t}\right)<0\right)>0 .
\end{aligned}
$$

The statement of Theorem 1 clarifies in what sense all intertemporal distortions are temporary in the second best. While there may be a strictly positive or negative wedge along the transition path, Theorem 1 implies that the wedge either converges to zero with probability one in the limit or alternates in sign with positive probability. Thus, at the limiting second best allocation, the intertemporal wedge is never strictly positive or strictly negative with probability one. For economies without aggregate shocks, Theorem 1 implies that $\beta F_{s s}^{k}=1$ at the steady state.

We prove Theorem 1 by decomposing the second best problem in two stages. In the first stage, we solve for the best admissible allocation taking as given the plan for the auxiliary variable. The second stage characterizes the optimal path for the auxiliary variable. Formally, let $\Psi^{A}(a) \subset \Psi^{F}$ be the set of feasible allocations such that the admissibility constraints (9) are satisfied at all nodes $s^{t} \in S^{t}, t \geq 0$ for given $a \in A^{\infty}$.

Definition 6 Let $W(a): A^{\infty} \rightarrow \Re$ be given by

$$
W(a)=\sup \left\{U\left(x ; s_{0}\right): \psi \in \Psi^{A}(a)\right\}
$$

By Assumption 3, the utility function $u_{i}$ is bounded above and below for all $i \in I$ as it is differentiable over a compact set. Thus $W(a)$ also is bounded above and below for all auxiliary variable plans. ${ }^{22}$ Clearly, if an admissible allocation $\psi^{*}$ is second best then there exists $a^{*} \in A^{\infty}$ such that

$$
W\left(a^{*}\right)=U\left(x^{*} ; s_{0}\right)
$$

and $\left\{\psi^{*}, a^{*}\right\}$ satisfy the admissibility constraints.

This decomposition of the second best problem imparts an intuitive structure to the proof of Theorem 1 and makes interpreting the results easier. In the first stage, we show that if all future admissibility constraints are non-binding, then all intertemporal distortions must be temporary. This result, derived in Proposition 1, establishes a link between permanent intertemporal distortions and the need to relax future binding admissibility constraints. Proposition 1 is very general, since the proof is based on a simple variational argument. Condition 1 is not used in this step of the proof.

\footnotetext{
${ }^{22}$ Technically, $\Psi^{A}(a)$ may be empty and then $W(a)=\varnothing$ but this case is clearly of no interest.
} 
The second stage characterizes the optimal path for the auxiliary variable and relies critically on Condition 1. Specifically, the sufficient condition connects the ability to front-load all distortions, which is a property of the set of admissible allocations, with the limiting behavior of the admissibility constraints at the optimum. Assumptions 5 and 7 also play an important role in the argument.

\subsection{First stage: Choosing allocations}

We start by analyzing the first-stage problem (14) given the plan $a^{*}$ for the auxiliary variable. Assumption 6 allows us to write the Lagrangian associated with the solution to (14) and derive the set of first order necessary conditions (f.o.n.c. henceforth). If there were no admissibility constraints at all, the f.o.n.c. would include the familiar Euler equation featuring a zero intertemporal wedge, $\Delta_{i}\left(s^{t}\right)=0$. In other words, if there are no constraints on the allocation of resources, the first order welfare effect of an additional unit of resources transferred from date $t$ to date $t+1$ at rate $F_{k, t+1}$ will have no first-order welfare effect. If the second best allocation displays a wedge, $\Delta_{i}\left(s^{t}\right) \neq 0$, transferring resources across periods tightens or relaxes future admissibility constraints, leading to a first order effect on welfare.

A key insight from the analysis of the first-stage problem is that the presence of intertemporal distortions is driven only by future binding admissibility constraints. Instead, current and past binding admissibility constraints, instead, are responsible for distortions in intratemporal margins, such as labor supply decisions. At the limiting second best allocation, the welfare effects associated with intratemporal distortions are, to a first-order approximation, symmetric across periods and do not generate a first-order welfare effect in connection to resource transfers across periods. Thus, we can rule out permanent intertemporal distortions if the admissibility constraints are not binding at future dates.

To formalize this reasoning, we define for each node a set of continuations at which all admissibility constrains are not binding. Given an allocation and auxiliary variable plan $\{\psi, a\}$, let

$$
\varpi_{m, i, t}=\left\{s^{t} \in S^{t} \mid \sup \left\{H_{m, i}\left(s^{j}\right)-a_{m, i}\left(s^{j}\right): s^{j} \in S^{j} \mid s^{t}, j \geq t\right\}<0\right\}
$$

and $\varpi_{m, t}=\cap_{I} \varpi_{i, m, t}, \varpi_{t}=\cap_{M} \varpi_{m, t}$ for all dates $t \geq 0 .{ }^{23}$ The set $\varpi_{t}$ collects all the nodes of $S^{t}$ such that no admissibility constraint is binding for all respective continuation nodes. The set $\varpi_{t}$ is a subset of $S^{t}$ with the property that $\varpi_{t} \subseteq \varpi_{t+1}$ at all dates. This property ensures that the probability sequence $\left\{\pi\left(s^{t} \in \varpi_{t}\right)\right\}$ is weakly increasing and converges.

We can now formally state the main result for the first stage of the proof.

Proposition 1 Let $\psi^{*}$ be a second best allocation, $\mu^{*}$ be the associated sequence of probability measures, and $a^{*}$ an auxiliary variable plan such that $\left\{\psi^{*}, a^{*}\right\}$ is admissible. If:

$$
\lim _{t \rightarrow \infty} \pi\left(s^{t} \in \varpi_{t}\right)=1
$$

\footnotetext{
${ }^{23}$ For ease of notation, we maintain the dependence of the set on the allocation $\psi$ and the auxiliary variable plan aimplicit.
} 
then for each $i \in I$ either the intertemporal distortion is zero on the limit with probability one,

$$
\mu_{\infty}^{*}\left(\Delta_{i}\left(s^{t}\right)=0\right)=1
$$

or the intertemporal distortion fluctuates around zero,

$$
\begin{aligned}
& \mu_{\infty}^{*}\left(\Delta_{i}\left(s^{t}\right)>0\right)>0 \\
& \mu_{\infty}^{*}\left(\Delta_{i}\left(s^{t}\right)<0\right)>0 .
\end{aligned}
$$

Proof. In the Appendix.

We will use a simplified model to illustrate the reasoning behind Proposition 1. Consider a second best economy with a representative agent, no uncertainty, and a single admissibility constraint for each date $t \geq 0$, given by:

$$
b\left(k_{t}\right)+\sum_{j=t}^{\infty} \beta^{j-t} h\left(c_{j}, l_{j}\right) \leq a_{t} .
$$

For this step of the proof, as in problem (14), the path for the auxiliary variable $\left\{a_{t}\right\}$ is taken as given. We will analyze the choice of the plan for the auxiliary variable in Section B.

To see why only future admissibility constraints distort the intertemporal margin, consider the first order welfare effect of transferring resources across periods. First, a marginal change in consumption at date $t$ may tighten certain admissibility constraints. To see this, assume $h_{t}^{c}>0$ and consider the marginal value of an extra unit of consumption at date $t$ :

$$
u_{t}^{c}=\frac{\lambda_{t}}{1+\frac{h_{t}^{c}}{u_{t}^{c}} \Phi_{t}} \leq \lambda_{t}
$$

where $\lambda_{t}, \lambda_{t+1}$, and $\phi_{t+1}$ are the Lagrangian multipliers associated to the resource constraint and the admissibility constraint (16) at $t$. The variable $\Phi_{t}=\sum_{j=0}^{t} \beta^{t-j} \phi_{j}$ collects the Lagrangian multipliers of all the admissibility constraints that feature the time $t$ allocation $\left\{c_{t}, l_{t}\right\}$. If the current or any past admissibility constraint are binding $\Phi_{t}>0$, there is a wedge between the marginal value of resources and the marginal utility of consumption. The wedge in (17) reflects the degree to which the allocation is distorted at that date. If the wedge at date $t+1$ is greater than the wedge at $t$,

$$
\frac{h_{t}^{c}}{u_{t}^{c}} \Phi_{t}<\frac{h_{t+1}^{c}}{u_{t+1}^{c}} \Phi_{t+1}
$$

a transfer of consumption from $t$ to $t+1$ will induce a first order welfare loss. At the steady state, this inequality will hold if $\Phi_{t}<\Phi_{t+1}$, that is, if the admissibility constraint at $t+1$ is binding.

In addition, changes in the capital stock may directly affect the admissibility constraint at date $t+1$. If the admissibility constraint is binding, there will be a wedge in the Euler equation for 
capital:

$$
\lambda_{t}+\phi_{t+1} b_{t+1}^{k}=\beta \lambda_{t+1} F_{t+1}^{k} .
$$

If $b_{t+1}^{k}>0$, additional capital tightens the admissibility constraint at $t+1$ and the wedge is positive.

Now consider a plan for the auxiliary variable such that the admissibility constraints from date $d$ onwards are not binding, so $\phi_{t}=0$ for all $t \geq d$. Evaluated at the steady-state, the wedge (17) between the marginal utility of consumption and the marginal value of resources reduces to:

$$
u_{s s}^{c}=\frac{\lambda_{s s}}{1+\frac{h_{s s}^{c}}{u_{s s}^{c}} \bar{\Phi}} \leq \lambda_{s s}
$$

where:

$$
\bar{\Phi}=\sum_{j=0}^{d} \beta^{d-j} \phi_{j} .
$$

Once all admissibility constraints stop binding, intratemporal distortions depend on the constant value, $\bar{\Phi}$, which captures the fact that allocations at date $t$ and $t+1$ are subject to the same set of admissibility constraints. Clearly, intratemporal distortions will persist in the long-run provided any admissibility constraint was binding at some date, since this implies $\bar{\Phi}>0$. However, at the steady state, equation (18) holds with equality, reflecting the fact that a shift in resources across dates has an offsetting first-order welfare effect on welfare. In addition, marginal changes in the level of capital cannot affect future admissibility constraints, since the constraints are not binding and the standard Euler equation holds:

$$
\lambda_{t}=\beta \lambda_{t+1} F_{t+1}^{k}
$$

The implication of this argument is that a transfer of resources across two consecutive periods has no first order welfare effects at the steady state if future admissibility constraints do not bind. It follows that the marginal value of resources is equated across periods and there are no intertemporal distortions at the steady state. This implies: $\beta F_{s s}^{k}=1$.

Proposition 1 characterizes the limiting behavior of the intertemporal wedge $\Delta_{i}\left(s^{t}\right)$, but it leaves open the possibility that the second best allocation features a strictly positive or negative wedge along the transition path. Since the stationary distribution may not be reached in finite time, a strictly positive or negative intertemporal wedge at all nodes would be implied. Corollary 1 shows that if the sign of the wedge $\Delta_{i}\left(s^{t}\right)$ is constant along the transition path, then the wedge is 0 with probability one in the limit.

Corollary 1 Let $\psi^{*}$ be a second best allocation, $\mu^{*}$ be the associated sequence of probability measures, and $a^{*}$ a plan for the auxiliary variable such that $\left\{\psi^{*}, a^{*}\right\}$ is admissible. In addition, let

$$
\lim _{t \rightarrow \infty} \pi\left(s^{t} \in \varpi_{t}\right)=1 \text {. }
$$


If for any $i \in I$, either: (i) $\Delta_{i}\left(s^{t}\right)>0$ for all $s^{t} \in S^{t}, t \geq 0$, or (ii) $\Delta_{i}\left(s^{t}\right)<0$ for all $s^{t} \in S^{t}, t \geq 0$, then:

$$
\mu_{\infty}^{*}\left(\Delta_{i}\left(s^{t}\right)=0\right)=1
$$

Proof. In the Appendix.

\subsection{Second stage: Choosing the auxiliary variable}

We now study the optimal choice of the plan for the auxiliary variable, which will denote with $a^{*}$. It is here that we use Condition 1 to show that the admissibility constraints will be binding only for a transitional phase. The argument proceeds in two steps. We first derive two important properties of the set of admissible plans for the auxiliary variable implied by Condition 1 . We then use these properties to show that the optimal plan for the auxiliary variable $a^{*}$ converges to a subset of $A$ where the admissibility constraints (9) do not bind.

Condition 1, combined with Assumption 5 and the compactness of $A$, can be used to derive two important properties of the set of admissible plans for the auxiliary variable. We illustrate the argument in the simplified model introduced in Section 4.1. We assume a simple formulation for the correspondence $\Gamma$ :

$$
\Gamma\left(a_{t}\right)=\left[0, \gamma\left(a_{t}\right)\right]
$$

where $\gamma$ is a function on $\Re_{+}$.

Let $\left\{x_{s s}^{f b}, k_{s s}^{f b}\right\}$ denote the steady-state under the first-best allocation. The sufficient condition is satisfied if there exists an admissible allocation such that $\left\{\hat{x}_{t}, \hat{k}_{t}\right\} \rightarrow\left\{x_{s s}^{f b}, k_{s s}^{f b}\right\}$. The allocation $\left\{\hat{x}_{t}, \hat{k}_{t}\right\}$ will, generally, not be optimal. However, its existence allows us to derive some key properties of the correspondence $\Gamma$ and of the set of admissible allocations.

Since $\left\{\hat{x}_{t}, \hat{k}_{t}\right\}$ is admissible, there must exist a path for the auxiliary variable, $\left\{\hat{a}_{t}\right\}$, that can support the first best steady state. This requires constraint (16) to be satisfied by the first best steady state allocation to satisfy:

$$
a_{s s}^{f b} \equiv b\left(k_{s s}^{f b}\right)+\frac{h\left(c_{s s}^{f b}, l_{s s}^{f b}\right)}{1-\beta} \leq \hat{a}_{t},
$$

for all dates $t \geq d$ for some date $d$. In addition, $\left\{\hat{a}_{t}\right\}$ will satisfy constraint (19) on the law of motion of the auxiliary variable.

The sequence $\left\{\hat{a}_{t}\right\}$ thus defines a subset of $A,\left\{a \geq a_{s s}^{f b}\right\}$, that supports the first-best steady state. It also identifies a path for the auxiliary variable that supports a first best continuation from any initial condition, since the admissibility constraints on the allocation, (16), and on the auxiliary variable, (19), must be satisfied at each date.

Proposition 2 formally establishes this result for the general environment. 
Proposition 2 Let:

$$
A^{f b}=\left\{\alpha \in A: \lim _{t \rightarrow \infty} \sup _{s^{j} \in S^{j}, j \geq t}\left\{H_{m, i}\left(\psi^{f b}, s^{j}\right)\right\} \leq \alpha_{m, i} \forall i, m\right\},
$$

where $\varphi^{f b}$ is the first best allocation. If Condition 1 holds, $A^{f b}$ is non-empty.

Proof. In the Appendix

Since $A^{f b}$ is non-empty, then by Assumption 5 for any $\alpha^{f b} \in A^{f b}$, the continuation plan ${ }_{t} \alpha^{f b}=$ $\left\{\alpha^{f b}, \alpha^{f b}, \ldots\right\}$ satisfies $\alpha^{f b} \in \Gamma\left(\alpha^{f b}, s^{j}\right) \forall s^{j} \in S^{j} \mid s^{t}$, for $j \geq t$. This property implies that for any $a \in A^{\infty}$ such that $a\left(s^{t}\right) \in A^{f b}$ for some $s^{t}$, then there is an admissible continuation plan for $a$ from $s^{t}$ such that every element is in $A^{f b}$. Thus, if $A^{f b}$ is non-empty, the first best stationary allocation can be supported by an admissible plan for the auxiliary variable.

We can now derive the second key property of the set of admissible plans for the auxiliary variable implied by Condition 1 . We will first illustrate this result in the simplified model. Here, the sufficient condition immediately rules out the possibility that for all $\alpha<a_{s s}^{f b}$ we have $\gamma(\alpha) \leq \alpha$. If this were the case, the existence of an auxiliary variable $\left\{\hat{a}_{t}\right\}$ such that $\hat{a}_{t} \geq a_{s s}^{f b}$ would be ruled out. Thus, the sufficient condition combined with Assumption 5, which requires $\Gamma(\cdot)$ to be convex and $\gamma(\alpha)$ to be concave, immediately implies $\gamma(\alpha)>\alpha$ for all $\alpha<a_{s s}^{f b}$. In other words, it is always possible to relax the admissibility constraints on the allocation in a one step continuation of the plan for the auxiliary variable.

This property is derived formally for the general framework in Proposition 3. It connects Condition 1, a global property of the set of admissible allocations, with a local property of the second best plan for the auxiliary variable.

Proposition 3 Let Condition 1 hold. Then, for any $\alpha \in A$, there exists a scalar $\varrho \in(0,1]$ and $\alpha^{\prime} \in A^{f b}$, such that:

$$
(1-\varrho) \alpha+\varrho \alpha^{\prime} \in \Gamma(\alpha, s)
$$

for some $s \in S$.

Proof. In the Appendix

If Proposition (21) did not hold, it would be possible to find a separating hyperplane between the set $\Gamma(a, s)$ for all $\{a, s\} \in A \times S$ and $A^{f b}$. Then, by Assumption 5 , the convexity of $\Gamma$ would imply that no point in the half-space containing $\Gamma(a, s)$ could be the starting point of a path leading to $A^{f b}$, contradicting Proposition 2 .

We now use these properties of the set of admissible plans for the auxiliary variables implied by Condition 1 to show that the second best plan converges to $A^{f b}$. The premise of this result is 
that, if at any date $t$ an admissibility constraint is binding, relaxing such a constraint has a first order welfare gain. For the simplified model, as long as an admissibility constraint is binding at date $t$, the optimal path for the auxiliary variable must feature $a_{t}^{*}=\gamma\left(a_{t-1}^{*}\right)>a_{t-1}^{*}$, that is, it must be strictly increasing. Thus, either the admissibility constraint will stop binding at some finite date $d$ or the auxiliary variable will converge to $a_{s s}^{f b}$. Our regularity conditions then ensure that if $a_{t}^{*} \rightarrow a_{s s}^{f b}$, the Lagrangian multiplier on the admissibility constraint $\phi_{t}$ converges to 0 .

The general framework features multiple admissibility constraints. Since there is no guarantee relaxing all of these simultaneously is possible, the plan for the auxiliary variable need not be monotone. However, Assumption 7 implies that an auxiliary variable plan $a$ can always be improved upon if it is in the interior of the image of the correspondence $\Gamma$. As in Ray (2002), this condition rules out local maxima and inflexion points and plays a crucial role in the argument.

Proposition 4 establishes this result formally for the general framework.

Proposition 4 Let Condition 1 hold and $\psi^{*}$ be a second best allocation such that $\mu_{\infty}^{*} \neq \mu_{\infty}^{f b}$. Then, there exists an auxiliary variable plan $a^{*}$ such that:

$$
\lim _{t \rightarrow \infty} \pi\left(s^{t} \in \varpi_{t}\right)=1
$$

and $\left\{\psi^{*}, a^{*}\right\}$ are admissible and $\varpi_{t}$ is defined in (15).

The requirement $\mu_{\infty}^{*} \neq \mu_{\infty}^{f b}$ restricts the attention to proper second best allocations, for which the limiting distribution does not converge to the first best.

\subsection{Combining the two stages}

Finally, we combine the results from both stages to prove Theorem 1. If the sufficient condition holds, by Proposition 4 it is possible to front load all distortions. Thus, the second best plan calls for eventually relaxing all future admissibility constraints. This implies that in the limit it is possible to transfer resources across periods without raising further distortions. By Proposition 1, it follows that permanent intertemporal distortions are not compatible with optimality.

\section{Applications}

We now discuss a series of application to illustrate the relevance of our result. Section 5.1 concentrates on Ramsey models and shows that the sufficient condition generally holds if the government can save enough. We also include two examples of Ramsey models with no permanent intertemporal distortions where Condition 1 does not hold. Section 5.2 examines economies with limited risk-sharing. In models with self-enforcement constraints, we show how the assumptions on the outside option determine whether the sufficient condition holds. For private information economies, we show that typically the sufficient condition does not hold, yet the optimal allocation still front-loads distortions. Finally, Section 5.3 analyzes economies where the government is subject to a limited 
commitment constraint. We show that when the government is benevolent, Condition 1 is always satisfied and there are no permanent intertemporal distortions. For a self-interested government, we discuss the conditions under which the sufficient condition holds.

\subsection{Ramsey Taxation with Asset Constraints}

The Ramsey taxation model rules out lump sum taxes. This restriction on fiscal instruments prevents the economy from attaining the first best allocation. But even without lump sum taxes, the first best steady state allocation can be attained if the government can save. By accumulating assets, the government eventually can finance spending with interest revenues and forgo distortionary taxes. Following this policy, though, requires maintaining a budget surplus for what could be a long transition phase and typically is not optimal. It does satisfy the admissibility constraints that characterize Ramsey policies. Hence, the sufficient condition holds and we can apply Theorem 1 . We now discuss a series of specific Ramsey models in order to show the link between the government's ability to save and permanent intertemporal distortions.

We begin with the basic Ramsey model with a representative agent and no uncertainty. The implementability constraint (1) is the only admissibility constraint, as discussed in Section 2.1. The sufficient condition holds if there exists an admissible allocation that converges to the first best steady state. This implies in turn that there exists a path for the auxiliary variable such that a first best continuation allocation is admissible from some date onwards.

Let $\bar{a}\left(k_{t}\right)$ denote the minimum value of the auxiliary variable that supports a first-best continuation allocation from date $t$ for $k_{t} \in K$. Evaluating constraint (1) at the first-best continuation allocation on any given date $t$ we obtain:

$$
\left(u_{t}^{c}\right)^{-1} \sum_{j=d}^{\infty} \beta^{j-t}\left(u_{j}^{c} c_{j}^{f b}+u_{j}^{l} l_{j}^{f b}\right)=\bar{a}\left(k_{t}\right) .
$$

An admissible allocation that converges to the first best steady-state is associated with a path for the auxiliary variable such that $a_{t} \geq \bar{a}\left(k_{t}\right)$ for some date $t .{ }^{24}$ We can derive the corresponding value of government debt, $\bar{b}\left(k_{t}\right)$, that sustains $\bar{a}\left(k_{t}\right)$ from the competitive equilibrium conditions:

$$
\bar{b}\left(k_{t}\right)=-\bar{a}\left(k_{t}\right)-F^{k}\left(k_{t}, l_{t}^{f b}\right) k_{t}
$$

The level $\bar{b}\left(k_{t}\right)$ will be negative, so a first best continuation allocation is admissible if the government can accumulate a sufficient level of assets. A policy with relatively high labor and capital income taxes for a transitional phase will lead to a level of debt such that $b_{t}<\bar{b}\left(k_{t}\right)$ at some date $t$, after which the first best continuation allocation from $k_{t}$ is admissible. Then, there will be no permanent intertemporal distortions, based on Theorem 1. In the basic Ramsey model, this also implies that the capital income tax is zero in the steady state.

Why would a government be unable to save? Assuming a closed economy, the government can

\footnotetext{
${ }^{24}$ To derive the full path of auxiliary variables we must write the full sequence of implementability constraints.
} 
save enough only if private agents can borrow enough. Denote with $b_{t}^{p}$ the debt issued by private agents. Bond market clearing requires $b_{t}^{p}+b_{t}=0$ at all dates. If a borrowing constraint is imposed on private agents, $b_{t}^{p} \leq B$, this gives rise to a corresponding upper limit on government assets, $b_{t} \geq-B$. This restriction will generate an additional admissibility constraint. ${ }^{25}$ If $\bar{b}\left(k_{t}\right)<-B$, the first-best continuation allocation cannot be supported from $k_{t}$. If $\bar{b}\left(k_{t}\right)<-B$ for all $k_{t} \in K$, then the sufficient condition is violated and the optimal allocation may feature intertemporal distortions.

While this example is very stylized, it carries a general lesson for Ramsey models. Borrowing constraints on the government will not lead to positive capital taxes. Saving constraints on the government and borrowing constraints on private agents may do so. This observation applies also to Ramsey policies with incomplete markets. Farhi (2007) analyzes a Ramsey model with a representative agent and risk-free debt. He finds that the limiting behavior of the second best allocation depends critically on the upper limit imposed on government assets. Following Aiyagari et al. (2002), debt and asset limits are labeled natural if they merely insure that obligations will be paid back almost surely. More stringent limits are referred to as ad hoc. ${ }^{26}$ Under the natural asset limit, the capital tax rate is either zero or fluctuates around zero, and there are no intertemporal distortions. ${ }^{27}$ Interpreting these findings in light of Theorem 1, the intertemporal wedge satisfies (13) and thus there are no permanent intertemporal distortions. Instead, under an ad hoc asset limit, the sufficient condition is not satisfied and the capital tax is typically different from zero.

We now turn to Ramsey models where Condition 1 is not satisfied. Perhaps the most interesting example is Aiyagari (1995). The economy features only idiosyncratic risk and markets are incomplete. Agents can accumulate capital and borrow subject to the constraint that their net worth is positive. There are no explicit borrowing or saving constraints on the government. Aiyagari (1995) shows that there are no permanent intertemporal distortions at the optimum. The intertemporal Euler equation for individual consumption satisfies (13). At the aggregate level, the condition $1=\beta F_{k}^{*}$ holds at the limiting distribution. The sufficient condition, though, is not satisfied. The first best allocation features full consumption insurance, which is not attainable given the restrictions on assets and fiscal instruments. Yet, the borrowing constraints on private agents do not impose constraints on aggregate capital. Moreover, private agents can borrow against their capital holdings as long as they maintain a positive net worth. As a result, the government can save enough to support the first best level of aggregate capital at the steady state. Thus, even if the sufficient condition is not satisfied, Aiyagari's results underscore the principle that the government's ability to save is critical for intertemporal distortions in a Ramsey model. ${ }^{28}$

\footnotetext{
${ }^{25}$ The additional constraint will shape the correspondence $\Gamma$ governing the path for the auxiliary variable.

${ }^{26}$ Aiyagari et al. (2002) derive the limiting properties of the Ramsey allocations in an incomplete markets model without capital. They show that, under the natural asset limit, if the process for aggregate shocks is ergodic, the economy converges almost surely to the first best steady state. If the process for aggregate shocks has an absorbing state, the limiting allocation coincides with the steady state of a Ramsey model with complete markets where the limiting value of taxes and debt depends on the value of the endogenous state variables at the time the absorbing state is reached. Under a more stringent asset limit, the economy may not converge.

${ }^{27}$ The properties of the intertemporal wedge vary with the assumptions on preferences and technology. Analytical results can be obtained only with quasi-linear preferences. Numerical simulations are used for general risk averse preferences.

${ }^{28}$ In Aiyagari (1995), the Ramsey allocation does not feature permanent intertemporal distortions but is imple-
} 
Finally, consider the standard Ramsey model under a balanced-budget constraint on the government. Since the government must finance spending solely from current tax revenues, it cannot save or borrow. Clearly, no admissible allocation converges to the first-best steady state. Since Condition 1 is sufficient but not necessary, there are versions of the balanced-budget Ramsey model that do not feature permanent intertemporal distortions. For example, if labor tax revenue is sufficient to finance government consumption, the steady state capital income tax is zero. ${ }^{29}$ However, the predictions on intertemporal distortions are not typically robust in this class of models. ${ }^{30}$

\section{$5.2 \quad$ Risk-Sharing}

We now discuss the presence of permanent intertemporal distortions in economies with idiosyncratic risk in light of Theorem 1. There are many frictions leading to incomplete risk-sharing. We revisit the two paradigms introduced in Section 2, models with self-enforcement constraints and models with private information. In economies with self-enforcement constraints, assumptions on the outside option have a crucial role. Because of the variety of formulations in the literature, there are contrasting implications for intertemporal distortions. We use Theorem 1 to clarify which features of the formulation are critical for intertemporal distortions in the long run. By contrast, private information economies typically exhibit permanent intertemporal distortions. As we will discuss, even if the sufficient condition does not hold in these economies, the front-loading principle still applies.

\subsubsection{Self-Enforcement Constraints}

Let us return to the example of an economy with self-enforcement constraints introduced in Section 2.2.1, extending it to allow for a general process for the productivity shocks, $\theta$.

We concentrate on the role of the outside option. There are a variety of formulations in the literature. These assumptions shape the resulting self-enforcement constraints and determine whether the sufficient condition is satisfied. The specification of the outside option necessarily involves the distribution of capital in case of default, as well as the agents' constraints after default.

We will consider two polar cases. The first assumes that a defaulting agent can seize a fraction $\delta \in[0,1]$ of the aggregate capital and operate it under autarchy. The resulting self-enforcement constraint is:

$$
\sum_{s=t}^{\infty} \beta^{s-t} \sum_{\theta^{s} \mid \theta^{t}} \pi_{\theta}\left(\theta^{s} \mid \theta^{t}\right) u\left(c_{i}\left(\theta^{s}\right), l_{i}\left(\theta^{s}\right) / \theta_{s}\right) \geq V_{\text {out }}\left(\delta k_{t} ; \theta_{t}\right),
$$

mented with a positive capital tax. This tax offsets the distortion on the intertemporal margin arising from the precautionary savings motive.

${ }^{29}$ See Judd (1985), Chamley (1986), as well as Stockman (2001).

${ }^{30}$ For example, Lansing (1999) shows that a zero capital tax is not optimal in a two-class economy with logarithmic preferences and a balanced-budget constraint. 
where $V_{\text {out }}$ satisfies:

$$
V_{\text {out }}\left(\delta k_{t} ; \theta_{t}\right)=\max _{\left\{x_{s}, \hat{k}_{s+1}\right\}_{s \geq t}} E_{\pi^{s} \mid \pi^{t}}\left[\sum_{s=t}^{\infty} \beta^{s-t} u\left(c_{s}, l_{s} / \theta_{s}\right)\right]
$$

subject to

$$
c_{s}+\hat{k}_{s+1} \leq F\left(\hat{k}_{s}, l_{s}\right)
$$

with $\hat{k}_{t}=\delta k_{t}$.

The value of the outside option, $V_{\text {out }}$, depends only on the level of aggregate capital at the time of default. The self-enforcement constraint can be relaxed by awarding utility in future periods, which is achieved via a back-loaded path of consumption. For a utilitarian social welfare function, the value of capital on the equilibrium path is greater than in the outside option, and the self-enforcement constraint is relaxed by raising capital. This property is preserved as long as the distribution of Pareto weights is not too uneven. As in Kocherlakota (1996), Condition 1 will be satisfied if the discount factor $\beta$ is high enough, agents' preferences exhibit a sufficient degree of risk aversion or the variance of the idiosyncratic shock is large enough.

The second case assumes that agents cannot accumulate capital after default or trade financial assets. Defaulting agents can still supply labor on competitive labor markets. Thus, the value of their outside option is:

$$
V_{\text {out }}\left(\left\{w_{s}\right\}_{s \geq t} ; \theta_{t}\right)=\max _{\left\{x_{s}\right\}_{s \geq t}} E_{\pi^{s} \mid \pi^{t}}\left[\sum_{s=t+1}^{\infty} \beta^{s-t} u\left(c_{s}, l_{s} / \theta_{s}\right)\right]
$$

subject to

$$
c_{s}^{i} \leq w_{s} l_{s}^{i}
$$

where $\left\{w_{s}\right\}_{s \geq t}$ is the sequence of future equilibrium wages.

The value of the outside option, through its dependence on wages, now involves the entire future path of capital. The resulting admissibility constraint does not comply with the general formulation in (9). More importantly, the dependence of the value of the outside option on the future path of capital generates a pecuniary externality. Since a rise in capital increases equilibrium wages and consumption for defaulting agents, capital in future periods need not relax the self-enforcement constraint. ${ }^{31}$ Even so, if agents are sufficiently patient, the first best allocation may satisfy the selfenforcement constraint. The impact of additional capital on incentives will depend on the degree of complementarity between labor and capital in production. This result is related to Davila, Hong, Krusell and Rios-Rull (2007).

\footnotetext{
${ }^{31}$ This formulation of the outside option hinges on the implicit assumption that aggregate capital cannot be contingent on default.
} 


\subsubsection{Examples}

\section{International Risk Sharing}

The international risk-sharing model of Kehoe and Perri $(2002,2004)$ is an interesting example of a self-enforcement constraint of the form (22). Each agent $i$ represents a country facing idiosyncratic total factor productivity shocks. The world resource constraint can be written as:

$$
\sum_{i=1,2}\left(c_{i, t}+k_{i, t+1}\right) \leq \sum_{i=1,2} \theta_{t}^{i}\left(k_{i, t}\right)^{\alpha}\left(l_{i, t}\right)^{1-\alpha}
$$

for all $t$. The formulation of the outside option implies that in case of default each country resorts to autarchy. The resulting self-enforcement constraint depends only on the value of capital at default and has the same structure as (22).

Kehoe and Perri (2002) show that the self-enforcement constraint generically distorts the Euler equation, since transferring consumption to a future period affects incentives. If the self-enforcement constraint is binding at $t+1$, there is a benefit from transferring consumption to time $t+1$ to relax this constraint. Specifically, reducing consumption at time $t$ tightens the self-enforcement constraint for periods $0-t$, while increasing consumption at $t+1$ relaxes all self-enforcement constraints for periods 0 to $t+1$. Thus, on net, transferring consumption to $t+1$ from $t$, relaxes more constraints than it tightens.

The sufficient condition is satisfied in this model if countries are weighted symmetrically in the world social welfare function. Based on Theorem 1, intertemporal distortions will be temporary.

\section{Ramsey taxation}

Chien and Lee (2008) study a Ramsey model with self-enforcement constraints. The government optimally sets linear taxes on labor and capital income. Defaulting agents supply labor on competitive markets and are subject to labor income taxes, but they cannot borrow or save. Crucially, the marginal tax rate on labor income cannot be conditional on default. It follows that the structure of the self-enforcement constraint is the same as in (23). They show that the limiting Ramsey equilibrium allocation does not feature intertemporal distortions, but the capital income tax is positive provided some self-enforcement constraints remain binding. This result resembles Aiyagari (1995), where instead of self-enforcement constraints, agents face arbitrary net worth constraints. In both environments there are no intertemporal distortions in the aggregate or in the individual allocation; however, the capital tax is positive to equate the intertemporal marginal rate of substitution across constrained and unconstrained agents.

\subsubsection{Private Information}

We now consider the same economy under the assumption that the idiosyncratic preference shocks, $\theta$, are private information. 
Generalizing (5), the incentive compatibility constraint is:

$$
\sum_{t=0}^{\infty} \beta^{t} \sum_{\theta^{t}} \pi_{\theta}\left(\theta^{t}\right) u\left(c_{i}\left(\theta^{t}\right), l_{i}\left(\theta^{t}\right) / \theta_{t}\right) \geq \sum_{t=0}^{\infty} \beta^{t} \sum_{\theta^{t}} \pi_{\theta}\left(\theta^{t}\right) u\left(c_{i}\left(\tilde{\theta}^{t}\right), l_{i}\left(\tilde{\theta}^{t}\right) / \theta_{t}\right)
$$

for all $\theta^{t}$ and $\tilde{\theta}^{t}$ in $\Theta^{t}$. Constraint (24) requires consumption to be spread out across states, in particular, consumption at time $t$ should be increasing in $\theta_{t}$ if the single crossing condition holds. Thus, the first best is not incentive compatible and Condition 1 does not hold. It is a well known result that, if utility is separable between consumption and labor, the second best allocation features a permanent intertemporal wedge. ${ }^{32}$ We can express this property as:

$$
u_{c}\left(c_{i}\left(\theta^{t}\right) ; \theta_{t}\right) \leq \beta F_{k} \sum_{\theta^{t+1} \mid \theta^{t}} \pi_{\theta}\left(\theta^{t+1} \mid \theta^{t}\right) u_{c}\left(c_{i}\left(\theta^{t+1}\right) ; \theta_{t+1}\right)
$$

where, with a slight abuse of notation, we use $u_{c}(c ; \theta)$ to denote the marginal utility of consumption for an agent with current type $\theta$. This inequality is strict when agents are risk averse and face idiosyncratic risk in the subsequent periods.

The incentive-compatibility constraint (24) is equivalent, with mild restrictions, to a sequence of incentive compatibility constraints over the continuation allocation. ${ }^{33}$ Adopting this formulation is useful for understanding the forces that give rise to the intertemporal wedge. Consider the government's trade-off in the allocation of consumption between two consecutive periods. If the future incentive compatibility constraint is binding, there is a shadow cost of transferring consumption to future periods. This cost reflects the fact that to preserve incentives, future consumption must be spread across states and will be worth less in terms of utility. Thus, an agent's optimal consumption allocation must be front-loaded. Since agents value a smooth consumption path, there is a tradeoff between smoothing consumption over time and minimizing the resource cost of a consumption allocation as long as future incentive compatibility constraints are binding.

Future incentive compatibility constraints will always be binding if agents are risk averse and face idiosyncratic shocks, leading to a permanent intertemporal distortion. If instead $\theta$ has an absorbing state that is reached with positive probability, intertemporal distortions will be temporary. For example, Werning (2007) examines an economy with fixed $\theta$ and shows that there are no permanent intertemporal distortions.

The front-loaded path of consumption under private information stands in contrast with the back-loaded path of consumption that arises with self-enforcement constraints of type (22). ${ }^{34}$ Despite this difference, admissibility constraints are relaxed over time and distortions are front-loaded in both environments. In the self-enforcement economy, promising more consumption in the future relaxes both the current and the future admissibility constraints. By contrast, in the private

\footnotetext{
${ }^{32}$ See Rogerson (1985) and Golosov, Kocherlakota and Tsyvinski (2003). Albanesi (2006) examines an economy with idiosyncratic capital income risk in which the intertemporal wedge has the opposite sign.

${ }^{33}$ See Spear and Srivastava (1987) and Green (1987).

${ }^{34}$ See Chapter 19 of Ljungqvist and Sargent (2004) for a discussion.
} 
information economy, awarding more consumption in the future relaxes the current incentive compatibility constraint but tightens future constraints. The front-loaded path of consumption is a way to relax future incentive compatibility constraints.

\subsection{Limited Commitment}

The government's policy choices may be constrained by a lack of commitment or by political economy considerations. These constraints often can be formulated in primal form and analyzed within our framework. A few examples of such environments are Fisher (1980), Chari and Kehoe (1990), Sleet and Yeltekin (2006b), Yared (2007), and Acemoglu, Golosov, and Tsyvinski (2008a, 2008b).

Theorem 1 can be used to derive a general result for models with a benevolent government with limited commitment. We show that Condition 1 is always satisfied when the government maximizes social welfare, so that limited commitment alone never leads to permanent intertemporal distortions. We then consider settings where the government is self-interested. Specifically, we analyze a simplified version of Acemoglu, Golosov, and Tsyvinski (2008a), where the allocation of resources is chosen by a rent-seeking ruler who has limited commitment. We discuss the determinants of intertemporal distortions in light of Theorem 1 and relate it to economies with self-enforcement constraints.

\subsubsection{Benevolent Government}

We start with the case of benevolent policymaker. Under limited commitment, an allocation $\psi$ is admissible if, for all $s^{t} \in S^{t}$ and $t \geq 0$, the continuation allocation $\psi \mid s^{t}$ is feasible from state $\left\{s^{t}, k\left(z^{t-1}\right)\right\}$, and is preferred to the outside option:

$$
U\left(x \mid s^{t} ; s^{t}\right) \geq V_{\text {out }}\left(x\left(s^{t}\right), k\left(z^{t-1}\right), s_{t}\right) .
$$

This admissibility constraint resembles the self-enforcement condition (22). Since the policymaker is benevolent, the continuation allocation is evaluated according to the social welfare function, $U$. The outside option $V_{\text {out }}(\cdot)$ may depend on the continuation allocation and the current state of the economy. A variety of specifications for the outside options have been considered. The most widely adopted is the notion of sustainable equilibrium developed by Chari and Kehoe (1990), where the outside option corresponds to a beneficial temporary deviation followed by a reversion to the worst subgame perfect equilibrium. For example, Reis (2006) analyzes a framework in which the worst sustainable equilibrium corresponds to financial autarchy for the government.

It is well known that the outside option plays a critical role in models with limited commitment, and it is hard to discriminate between the many possible specifications. However, since any outside

option must be feasible and the first best continuation allocation from state $\left\{s^{t}, k\left(z^{t-1}\right)\right\}$ satisfies 
(8), it follows that: ${ }^{35}$

$$
U\left(x^{f b} \mid\left\{s^{t}, k\left(z^{t-1}\right)\right\} ; s^{t}\right) \geq V_{\text {out }}\left(x\left(s^{t}\right), k\left(z^{t-1}\right), s_{t}\right) .
$$

Thus, there exists an admissible allocation that converges to the first best limiting allocation and Condition 1 is satisfied.

The application of Theorem 1 clarifies that limited commitment alone will not give rise to permanent intertemporal distortions and that this is a robust property of this class of second best economies. Limited commitment may give rise to permanent intertemporal distortions in combination with other frictions. Chari and Kehoe (1990) and Phelan and Stacchetti (2001) study sustainable equilibria in an economy where the government is subject to a balanced budget constraint. Since the government is unable to save, the limiting first best allocation is not admissible and the sufficient condition is violated, as discussed in Section 5.1. Then, sustainable equilibria can display permanent intertemporal distortions.

\subsubsection{Self-Interested Policymaker}

We now turn to environments where the policymaker is not benevolent. As an example, we consider a simplified version of the economy in Acemoglu, Golosov and Tsyvinski (2008a, 2008b) with no aggregate or idiosyncratic risk. The economy is populated by two types of agents. A continuum of identical private agents value streams of consumption and labor, according to standard preferences. In addition, a rent-seeking ruler values streams of transfers $\left\{T_{t}\right\}_{t \geq 0}$ according to the utility function:

$$
\sum_{t=0}^{\infty} \beta^{t} v\left(T_{t}\right)
$$

where $v(\cdot)$ is strictly increasing and concave with $v(0)=0$ and $\beta \in(0,1)$.

The social welfare function for this economy can be written as:

$$
\sum_{t=0}^{\infty} \beta^{t} u\left(c_{t}, l_{t}\right)+\gamma \sum_{t=0}^{\infty} \beta^{t} v\left(T_{t}\right),
$$

allowing a positive Pareto weight, $\gamma \geq 0$, for the ruler. This formulation clarifies the conflict over the distribution of resources between private agents and the ruler.

The ruler can capture a fraction of aggregate output by resorting to expropriation. Thus, any admissible allocation must satisfy:

$$
\sum_{j \geq 0} \beta^{j} v\left(T_{t+j}\right) \geq v\left(\kappa F\left(k_{t}, l_{t}\right)\right)
$$

where the parameter $\kappa$, intended to capture the quality of political institutions in the model, rep-

\footnotetext{
${ }^{35}$ Indeed, if $U\left(x \mid s^{t} ; s^{t}\right)<V_{\text {out }}\left(x\left(s^{t}\right), k\left(z^{t-1}\right), s_{t}\right)$ then the set of admissible allocations is empty.
} 
resents the fraction of aggregate output that the ruler can extract. ${ }^{36}$ This admissibility constraint has the same structure as the self-enforcement condition in (22).

Is it possible to find an allocation that satisfies (27) and converges to the first best? In the first best steady state, the ruler is awarded a constant share of output and her consumption is constant. As in models with self-enforcement constraints, the first best steady-state is admissible if the discount factor is high enough, for a given $\kappa$. It is also admissible if $\kappa$ is low enough. In particular, if $\kappa=0$ the admissibility constraint is trivially satisfied and the limited commitment problem disappears. Finally, the first best steady state is admissible if the ruler's weight in the social welfare function, $\gamma$, is large enough. Thus, even if the policymaker is not benevolent, limited commitment will not lead to permanent intertemporal distortions as long as the conflict over the distribution of resources is not too severe.

Acemoglu, Golosov and Tsyvinski (2008a) also allow the ruler to have a different intertemporal discount factor, $\delta$, and show that for $\delta \geq \beta$, there are no intertemporal distortions in the limit. ${ }^{37}$ While our framework does not allow for heterogeneity in discount factors, it is still possible to relate this result to Condition $1 .{ }^{38}$ For $\delta \geq \beta$, the first best allocation specifies a growing consumption share for the ruler. The first best allocation must then satisfy the admissibility constraint after a finite number of periods, and it easy to see that it will always be possible to find a transition path that is admissible and converges to the first best steady state.

\section{Conclusion}

Theorem 1 clarifies that in second best economies, permanent intertemporal distortions arise only if future admissibility constraints are binding. Our unified approach is critical for this insight, since it allows us to identify an optimality principle that is common across a broad class of environments. In all of these economies, the optimal allocation front-loads distortions. If our sufficient condition holds, all distortions can be front-loaded. The implication here is that intertemporal distortions will be temporary since eventually, no future admissibility constraints will bind. On the other hand, since intratemporal distortions depend on the path of past binding constraints, these typically will persist in the long run.

A natural question is how applicable the logic of Theorem 1 is to environments outside our general framework. Our argument requires restrictions on the degree of history dependence in the admissibility constraints. In Section 5.2, we discussed an economy with self-enforcement constraints in which this restriction is not satisfied, where the general logic still applies. However, it is hard to derive general conditions for the validity of the front-loading principle without placing restrictions on history dependence of the admissibility constraints.

Our analysis presumes the existence of a second best plan that weakly converges to a limiting stationary allocation. Interestingly, in many economies that feature permanent intertemporal dis-

\footnotetext{
${ }^{36}$ Following expropriation, the ruler looses power and obtains no transfers.

${ }^{37}$ See also Sleet and Yeltekin (2006b).

${ }^{38}$ In addition, the constrained efficient allocation may not satisfy the interiority assumptions we have made.
} 
tortions, the second best allocation does not converge. Private information economies are a notable example. ${ }^{39}$ It is well known that imposing a lower bound on continuation utility, as in Atkeson and Lucas (1995), or allowing the government to discount the future at a lower rate than do private agents, ${ }^{40}$ as in Phelan (2006) and Farhi and Werning (2007), will guarantee the existence of a nondegenerate limiting distribution. Yet, the optimal allocation still features permanent intertemporal distortions, suggesting that for this class of economies, the presence of intertemporal distortions does not depend on the convergence properties of the optimal allocation. By contrast, in the Ramsey model with incomplete markets analyzed by Farhi (2007), when an ad hoc asset limit is binding and the sufficient condition does not hold, the Ramsey allocation does not converge and intertemporal distortions are permanent. An analysis of the link between the convergence properties of the second best allocation and the presence of intertemporal distortions would be of clear interest. We leave this topic for future work.

Finally, we consider only infinite-horizon economies. Erosa and Gervais (2002), Garriga (2003), and Krueger, Conesa, and Kitao (2008) analyze overlapping generation Ramsey models where it may be optimal to tax capital. Overlapping generations economies often can display dynamic inefficiencies, possibly providing a rationale for capital taxation absent from infinite horizon Ramsey models. In addition, with overlapping generations, shifting distortions over time entails a transfer across generations. A unified analysis of intertemporal distortions in overlapping generations and infinite-horizon economies would be very interesting to pursue, but is beyond the scope of this paper. A more extensive discussion of the determinants of intertemporal distortions in overlapping generations is available in Albanesi and Armenter (2007).

\section{References}

[1] Acemoglu, Daron, Mikhail Golosov, and Aleh Tsyvinski. 2008a. Political Economy of Mechanisms. Econometrica, 76(3): 619-641.

[2] Acemoglu, Daron, Mikhail Golosov, and Aleh Tsyvinski. 2008b. Market versus Governments. Journal of Monetary Economics, 55(1): 159-189.

[3] Aiyagari, S. Rao. 1995. Optimal Capital Income Taxation with Incomplete Markets, Borrowing Constraints, and Constant Discounting. Journal of Political Economy 103(6): 1158-75,

[4] Aiyagari, S. Rao, Albert Marcet, Thomas J. Sargent, and Juha Seppala. 2002. Optimal Taxation without State-Contingent Debt. Journal of Political Economy 110 (6): 1220-1254.

[5] Albanesi, Stefania. 2006. Optimal Taxation of Entrepreneurial Capital with Private Information. NBER WP 12419.

\footnotetext{
${ }^{39}$ See Green (1987), Thomas and Worral (1990), Atkeson and Lucas (1992) and Phelan (1998).

${ }^{40}$ Sleet and Yeltekin (2006a) show that limited commitment can provide a rationale for such differential discounting.
} 
[6] Albanesi, Stefania. 2008.Discussion of "Market versus Governments." Journal of Monetary Economics 55 (1).

[7] Albanesi, Stefania and Roc Armenter. 2007. Understanding Capital Taxation in Ramsey Models. Working paper, Federal Reserve Bank of New York.

[8] Albanesi, Stefania and Christopher Sleet. 2006. Dynamic Optimal Taxation with Private Information. The Review of Economic Studies, 73: 1-30.

[9] Armenter, Roc. 2008. A Note on Incomplete Factor Taxation. Journal of Public Econmics 92 (10-11): 2275-2281.

[10] Atkeson, Andrew and Robert E. Lucas, Jr. 1993. On Efficient Distribution with Private Information. Review of Economic Studies 59: 427-453.

[11] Atkeson, Andrew and Robert E. Lucas, Jr. 1995. Efficiency and Equality in a Simple Model of Unemployment Insurance. Journal of Economic Theory 66 (1): 64-88.

[12] Atkeson, Andrew, V.V. Chari, and Patrick J. Kehoe. 1999. Taxing Capital Income: A Bad Idea. Federal Reserve Bank of Minneapolis Quarterly Review 23 (3): 3-17.

[13] Atkinson, Anthony B. and Joseph E. Stiglitz. 1980. Lectures on Public Economics. McGrawHill, New York.

[14] Chamley, Christophe, 1986. Optimal Taxation of Capital Income in General Equilibrium with Infinite Lives. Econometrica 54 (3): 607-622.

[15] Chari, V.V., and Patrick J. Kehoe. 1990. Sustainable Plans. Journal of Political Economy, 98 (4): 783-802.

[16] Chari, V.V., and Patrick J. Kehoe. 1999. Optimal fiscal and monetary policy. In J. B. Taylor, and M. Woodford (ed.), Handbook of Macroeconomics.

[17] Chen, Yili and Junsang Lee. 2008. Why Tax Capital? Working paper, Purdue University.

[18] Conesa, Juan Carlos, Sagiri Kitao, and Dirk Krueger. 2008. Taxing Capital? Not a Bad Idea After All! American Economic Review, forthcoming.

[19] Correia, Isabel H. 1996. Should Capital Income Be Taxed in the Steady State? Journal of Public Economics 60 (1): 147-151.

[20] Davila, J., J. Hong, P. Krusell and V. Rios-Rull. 2007. Constrained-Efficient Allocations in the Growth Model with Idiosyncratic Shocks. Working paper, University of Pennsylvania.

[21] Diamond, P. and Mirrlees, J. 1978. A model of social insurance with variable retirement. Journal of Public Economics 10: 295-336. 
[22] Erosa, Andres and Martin Gervais. 2002. Optimal Taxation in Life-Cycle Economies. Journal of Economic Theory 105(2): 338-369.

[23] Farhi, Emmanuel. 2007. Capital Taxation and Ownership When Markets are Incomplete. NBER WP 13390.

[24] Farhi, Emmanuel and Ivan Werning. 2007. Inequality and Social Discounting. Journal of Political Economy 115: 365-402.

[25] Fischer, Stanley. 1980. Dynamic Inconsistency, Cooperation, and the Benevolent Dissembling Government. Journal of Economic Dynamics and Control 2: 93-107.

[26] Garriga, Carlos. 2003. Optimal Fiscal Policy in Overlapping Generation Models. Manuscript. Florida International University.

[27] Golosov, Mikhail Narayana Kocherlakota, Aleh Tsyvinski. 2003. Optimal Indirect and Capital Taxation. The Review of Economic Studies, Volume 70, Issue 3, Page 569.

[28] Jones, Larry E., Rodolfo E. Manuelli, and Peter E. Rossi. 1997. On the Optimal Taxation of Capital Income. Journal of Economic Theory 73(1): 93-117.

[29] Judd, Kenneth. 1985. Redistributive Taxation in a Perfect Foresight Model. Journal of Public Economics 28, 59-84.

[30] Kehoe, Patrick and Fabrizio Perri. 2002. International Business Cycles with Endogenous Incomplete Market. Econometrica 70 (3): 907-928.

[31] Kehoe, Patrick and Fabrizio Perri. 2004. Competitive Equilibria with Limited Enforcement. Journal of Economic Theory 119 (1): 184-206.

[32] Kocherlakota, Narayana. 1996. Implications of Efficient Risk Sharing without Commitment. Review of Economic Studies 63: 595-610.

[33] Kocherlakota, Narayana. 2005a. Advances in Dynamic Optimal Taxation. Paper presented at the World Congress of the Econometric Society, August 2005.

[34] Kocherlakota, Narayana. 2005b. Zero Expected Wealth Taxes: A Mirrlees Approach to Dynamic Optimal Taxation. Econometrica.

[35] Lansing, Kevin. 1999. Optimal Redistributive capital Taxation in a Neoclassical Growth model. Journal of Public Economics 73, 423-453.

[36] Ljungqvist, Lars and Thomas J. Sargent. 2004. Recursive Macroeconomic Theory. The MIT Press.

[37] Lucas, Robert E., Jr., and Nancy L. Stokey. 1983. Optimal Fiscal and Monetary Policy in an Economy without Capital. Journal of Monetary Economics 12: 55-93. 
[38] Mirrlees, James. 1971. An exploration in the theory of optimum income taxation. The Review of Economic Studies 38: 175-208.

[39] Phelan, Christopher. 1998. On the Long-Run Implications of Repeated Moral Hazard. Journal of Economic Theory, 79(2): 174-191.

[40] Phelan, Christopher. 2006. Opportunity and Social Mobility. The Review of Economic Studies 73: 487-504.

[41] Phelan, Christopher and Ennio Stacchetti. 2001. Econometrica, 69(6): 1491-1518.

[42] Ray, Debraj. The Time Structure of Self-Enforcing Agreements. Econometrica 70 (2), 547-582.

[43] Reis, Catarina. 2006. Taxation without Commitment. Manuscript, MIT.

[44] Rogerson, William. 1985. Repeated moral hazard. Econometrica 53:69-76.

[45] Salanie, Bernard. 2003. The economics of Taxation. The MIT Press, Cambridge, MA.

[46] Sleet, Christopher and Sevin Yeltekin. 2006a. Credibility and Endogenous Societal Discounting. Review of Economic Dynamics, 9 (3): 410-437.

[47] Sleet, Christopher and Sevin Yeltekin. 2006b. Optimal Taxation with Endogenously Incomplete Debt Markets. 2006. Journal of Economic Theory, 127: 36-73.

[48] Spear, Stephen and Sanjay Srivastava. 1987. On Repeated Moral Hazard with Discounting. Review of Economic Studies 54 (5): 599-617.

[49] Stokey, Nancy and Robert Lucas. 1996. Recursive Methods in Economic Dynamics. Harvard University Press, Cambridge, MA.

[50] Stockman, David. 2001. Balanced-Budget Rules: Welfare Loss and Optimal Policies. Review of Economic Dynamics 4, 438-459.

[51] Thomas, Jonathan and Tim Worral. 1990. Income Fluctuation and Asymmetric Information. Journal of Economic Theory 51 (2): 367-390.

[52] Tuomala, Matti. 1990. Optimal Income Tax and Redistribution. Claredon Press, Oxford, UK.

[53] Werning, Ivan. 2007. Optimal Fiscal Policy with Redistribution. The Quarterly Journal of Economics CXXII.

[54] Yared, Pierre. 2007. Politicians, Taxes and Debt. Manuscript, MIT.

[55] Zhu, Xiaodong. 1992. Optimal Fiscal Policy in a Stochastic Growth Model. Journal of Economic Theory. 58: 250-89. 


\section{A Proofs for Stage 1}

\section{A.1 Proof of Proposition 1}

The set of plans $\Psi$ is a complete vector space with the sup norm. $U, F$, and all terms in $H$ are real-valued and twice differentiable functions by Assumptions 2, 3, and 4. Let $\psi^{*}$ be a second best plan and $a^{*}$ an auxiliary variable plan such that $\left\{\psi^{*}, a^{*}\right\}$ is admissible. By Assumption $6, \psi^{*}$ is a regular point of all the binding constraints in Problem 14 given $a^{*}$. All conditions are satisfied thus to apply the generalized Kuhn-Tucker theorem (See Luenberger (1997), page 294) and characterize the solution $\psi^{*}$ with the Lagrangian

$$
\begin{aligned}
\Lambda(\psi ; \varphi, \phi, \lambda)= & U\left(x ; s_{0}\right) \\
& -\sum_{t=0}^{\infty} \sum_{z^{t} \in Z^{t}} \beta^{t} \pi\left(z^{t} \mid z_{0}\right) \lambda\left(z^{t}\right)\left\{\mathbf{c}\left(z^{t}\right)+k\left(z^{t}\right)-F\left(z^{t}\right)\right\} \\
& -\sum_{t=0}^{\infty} \sum_{z^{t} \in Z^{t}} \beta^{t} \pi\left(z^{t} \mid z_{0}\right) \varphi\left(z^{t}\right)\left\{\sum_{i \in I} \sum_{\theta^{t} \in \Theta^{t}} \pi_{i} \pi_{\theta}\left(\theta^{t}\right) y_{i}\left(z^{t}, \theta^{t}\right)-k\left(z^{t}\right)\right\} \\
& -\sum_{t=0}^{\infty} \sum_{s^{t} \in S^{t}} \beta^{t} \pi\left(s^{t} \mid s_{0}\right) \phi\left(s^{t}\right)^{\prime}\left\{H\left(s^{t}\right)-a\left(s^{t}\right)\right\},
\end{aligned}
$$

where the dependence of $H$ and $F$ on $\psi$ is understood. The Lagrangian multipliers $\phi\left(s^{t}\right)$ are stacked vectors of length $I \times M$.

If $s^{d} \in \varpi_{d}$ then $s^{t} \in \varpi_{t}$ for all $s^{t} \in S^{t} \mid s^{d}$. By definition,

$$
H_{m, i}\left(s^{t}\right)-a_{m, i}\left(s^{t}\right) \leq \sup \left\{H_{m, i}\left(s^{j}\right)-a_{m, i}\left(s^{j}\right): s^{j} \in S^{j} \mid s^{t}, j \geq t\right\}<0
$$

and the Kuhn-Tucker conditions associated with the Lagrangian imply that $\phi_{m, i}\left(s^{t}\right)=0$ for all $s^{t} \in S^{t} \mid s^{d}$ and all $\{i, m\} \in I \times M$.

Next we write the first order necessary conditions (f.o.n.c.) associated with $x\left(s^{t}\right)$ for any node $s^{t} \in S^{t} \mid s^{d}, t>d+1$ for some $s^{d} \in \varpi_{d}$. For $c_{i}\left(s^{t}\right)$ we have that

$$
\begin{aligned}
\pi_{i}\left(\beta^{t} \pi\left(s^{t}\right)\right)\left(u_{i}^{c}\left(s^{t}\right)-\lambda\left(z^{t}\right)\right)= & \beta^{t} \pi\left(s^{t}\right)\left(\sum_{j=0}^{d} \phi\left(s^{j}\right) \cdot d_{i}\left(s^{j}\right)\right)^{\prime}\left[\frac{\partial h_{m, i}^{0}\left(x\left(s^{t}\right) ; s_{t}\right)}{\partial c_{i}\left(s^{t}\right)}\right]_{m}+ \\
& \left(\beta^{t} \sum_{j=0}^{d} \sum_{\tilde{s}^{j} \in S^{j} \mid s^{t-1}} \pi\left(s^{t} \mid s^{j}\right) \pi\left(\tilde{s}^{j} \mid s_{0}\right) \phi_{i}\left(\tilde{s}^{j}\right) \cdot d_{i}\left(\tilde{s}^{j}\right)\right)^{\prime}\left[\frac{\partial h_{m, i}^{1}\left(x\left(s^{t}\right) ; s_{t}\right)}{\partial c_{i}\left(s^{t}\right)}\right]_{m}
\end{aligned}
$$

where $\phi_{i}\left(s^{j}\right)$ is a vector collecting the $M$ Lagrangian multipliers associated with the admissibility constraints of ex-ante type $i$ at node $s^{j} ; d_{i}\left(s^{j}\right)$ and $\left[\frac{\partial h_{m, i}^{0}\left(x\left(s^{t}\right) ; s_{t}\right)}{\partial c_{i}\left(s^{t}\right)}\right]_{m}$ are vectors similarly stacked. 
We have used Assumption 6 to write the f.o.n.c. with strict equality. We simplify the term

$$
\beta^{t} \sum_{j=0}^{d} \sum_{\tilde{s}^{j} \in S^{j} \mid s^{t-1}} \pi\left(s^{t} \mid s^{j}\right) \pi\left(\tilde{s}^{j} \mid s_{0}\right) \phi_{i}\left(\tilde{s}^{j}\right) \cdot d_{i}\left(\tilde{s}^{j}\right)=\beta^{t} \pi\left(s^{t}\right) \sum_{j=0}^{d} \sum_{\tilde{s}^{j} \in S^{j} \mid s^{t-1}} \frac{\pi\left(\tilde{s}^{j} \mid s_{0}\right)}{\pi\left(s^{j} \mid s_{0}\right)} \phi_{i}\left(\tilde{s}^{j}\right) \cdot d_{i}\left(\tilde{s}^{j}\right) .
$$

By Assumption 1 the term is finite. Rewrite the f.o.n.c. as

$$
\pi_{i} u_{i}^{c}\left(s^{t}\right)-\pi_{i} \lambda\left(z^{t}\right)=\left[\bar{\phi}_{i}^{0}\left(s^{d}\right)\right]^{\prime}\left[\frac{\partial h_{m, i}^{0}\left(x\left(s^{t}\right) ; s_{t}\right)}{\partial c_{i}\left(s^{t}\right)}\right]_{m}+\left[\bar{\phi}_{i}^{1}\left(s^{d}\right)\right]^{\prime}\left[\frac{\partial h_{m, i}^{1}\left(x\left(s^{t}\right) ; s_{t}\right)}{\partial c_{i}\left(s^{t}\right)}\right]_{m}
$$

where

$$
\begin{aligned}
\bar{\phi}_{i}^{0}\left(s^{d}\right) & =\left(\sum_{j=0}^{d} \phi_{i}\left(s^{j}\right) \cdot d_{i}\left(s^{j}\right)\right) \\
\bar{\phi}_{i}^{1}\left(s^{d}\right) & =\left(\sum_{j=0}^{d} \sum_{\tilde{s}^{j} \in S^{j} \mid s^{t-1}} \frac{\pi\left(\tilde{s}^{j} \mid s_{0}\right)}{\pi\left(s^{j} \mid s_{0}\right)} \phi_{i}\left(\tilde{s}^{j}\right) \cdot d_{i}\left(\tilde{s}^{j}\right)\right) .
\end{aligned}
$$

Similarly, the f.o.n.c. for labor $l_{i}\left(s^{t}\right)$ for nodes $s^{t} \in S^{t} \mid s^{d}$ can be written as

$\pi_{i} u_{i}^{l}\left(s^{t}\right)+\pi_{i} \lambda\left(z^{t}\right) F^{l}\left(z^{t}\right)=\left[\bar{\phi}_{i}^{0}\left(s^{d}\right)\right]^{\prime}\left[\frac{\partial h_{m, i}^{0}\left(x\left(s^{t}\right) ; s_{t}\right)}{\partial l_{i}\left(s^{t}\right)}\right]_{m \leq M}+\left[\bar{\phi}_{i}^{1}\left(s^{d}\right)\right]^{\prime}\left[\frac{\partial h_{m, i}^{1}\left(x\left(s^{t}\right) ; s_{t}\right)}{\partial l_{i}\left(s^{t}\right)}\right]_{m \leq M}$.

Finally the first order for aggregate capital $k\left(z^{t}\right), z^{t} \in Z^{t} \mid z^{d}$, is

$$
\lambda\left(z^{t}\right)=\beta \sum_{z^{t+1} \in Z^{t+1} \mid z^{t}} \pi_{z}\left(z_{t+1} \mid z_{t}\right) \lambda\left(z^{t+1}\right) F^{k}\left(z^{t+1}\right) .
$$

The system of equations given by the f.o.n.c. for consumption $c_{i}\left(s^{t}\right)$, labor $l_{i}\left(s^{t}\right)$ and capital $k\left(z^{t}\right)$ (28)-(30), and the resource constraint (6) is a function of the aggregate shock $z_{t}$, the realization of the idiosyncratic shock $\theta_{t}$, the stock of aggregate capital $k\left(z^{t-1}\right)$, and the constants $\left\{\bar{\phi}_{i}^{0}, \bar{\phi}_{i}^{1}\right\} \equiv$ $\left\{\bar{\phi}_{i}^{0}\left(s^{d}\right), \bar{\phi}_{i}^{1}\left(s^{d}\right)\right\}$. Thus, we can express $\left\{x_{i}\left(s^{t}\right), k\left(z^{t}\right)\right\}$ as:

$$
\begin{aligned}
x_{i}\left(s^{t}\right) & =f_{x}\left(\sigma ; \bar{\phi}_{i}^{0}, \bar{\phi}_{i}^{1}\right), \\
k\left(z^{t}\right) & =f_{k}\left(\sigma ; \bar{\phi}_{i}^{0}, \bar{\phi}_{i}^{1}\right),
\end{aligned}
$$

for all $s^{t} \in S^{t} \mid s^{d}$, where

$$
\sigma=\left\{s^{t}, k\left(z^{t-1}\right)\right\} \in \Sigma \equiv S \times K
$$

and

$$
\begin{array}{ll}
f_{x}: & \Sigma \rightarrow X \\
f_{k} & : \quad \Sigma \rightarrow K .
\end{array}
$$


The dependence on the constants $\left\{\bar{\phi}_{i}^{0}, \bar{\phi}_{i}^{1}\right\}$ will henceforth be omitted for ease of notation. The functions $f_{x}$ and $f_{k}$ do not describe all the allocations in the economy, but only those in continuations nodes of a given $s^{d} \in \varpi_{d}$. Under Assumptions 2, 3, and 4, both $f_{x}$ and $f_{k}$ are bounded.

Let $\Omega_{i}(\sigma): \Sigma \rightarrow \Re$ be given by:

$$
\Omega_{i}(\sigma) \equiv 1-\frac{1}{u_{i}^{c}(\sigma)}\left(\left(\bar{\phi}_{i}^{0}\right)^{\prime}\left[\frac{\partial h_{m}^{0}(\sigma)}{\partial c_{i}}\right]_{m \leq M}+\left(\bar{\phi}_{i}^{1}\right)^{\prime}\left[\frac{\partial h_{m}^{1}(\sigma)}{\partial c_{i}}\right]_{m \leq M}\right)
$$

for some $i \in I$, where we have written $u_{i}^{c}(\sigma)$ for $u_{i}^{c}\left(s^{t}\right)$ and so on. Moreover, Assumptions 3 and 4 implies that first derivatives of $u_{i}, h_{m}^{0}$, and $h_{m}^{1}$ must be bounded above and below in $[0, \bar{c}] \times[0, \bar{l}]$, and $u_{i}^{c}$ is strictly positive. It follows that for all $\sigma \in \Sigma, \Omega_{i}(\sigma)$ is bounded above and below.

Define the operator $\Xi$ on bounded functions as:

$$
\Xi\left[\Omega_{i}\right](\sigma)=\frac{\sum_{s^{\prime} \in S} \omega_{i}\left(\sigma, s^{\prime}\right) \Omega_{i}\left(\sigma^{\prime}\right)}{\sum_{s^{\prime} \in S} \omega_{i}\left(\sigma, s^{\prime}\right)}
$$

where

$$
\omega_{i}\left(\sigma, s^{\prime}\right)=\beta \pi\left(s^{\prime} \mid s\right) u_{i}^{c}\left(\sigma^{\prime}\right) F^{k}\left(\sigma^{\prime}\right)
$$

and $\sigma^{\prime}$ is given by

$$
\sigma^{\prime}=\left\{s^{\prime}, f_{k}(\sigma)\right\}
$$

for each $s^{\prime} \in S$. By construction,

$$
u_{i}^{c}\left(s^{t}\right) \leq(\geq) \beta \sum \pi\left(s^{t+1} \mid s_{t}\right) u_{i}^{c}\left(s^{t+1}\right) F^{k}\left(s^{t+1}\right)
$$

if and only if:

$$
\Xi\left[\Omega_{i}\right](\sigma) \leq(\geq) \Omega_{i}(\sigma)
$$

By Assumptions 1 and 8, there must exist an an ergodic set $\Sigma_{\infty} \subseteq \Sigma$. That is, if $\sigma \in \Sigma_{\infty}$ then $\sigma^{\prime}=\left\{s^{\prime}, f_{k}(\sigma)\right\} \in \Sigma_{\infty}$ for all realizations of $s^{\prime} \in S$. We derive the two key properties of operator $\Omega$ in the following Lemma.

Lemma 1 Let $\Sigma_{\infty}$ be an ergodic set. If for all $\sigma \in \Sigma_{\infty}$ :

$$
\Xi\left[\Omega_{i}\right](\sigma) \geq \Omega_{i}(\sigma)
$$

then,

$$
\Xi\left[\Omega_{i}\right](\sigma)=\Omega_{i}(\sigma),
$$

for all $\sigma \in \Sigma_{\infty}$.

If , instead, for all $\sigma \in \Sigma_{\infty}$ :

$$
\Xi\left[\Omega_{i}\right](\sigma) \leq \Omega_{i}(\sigma),
$$


then,

$$
\Xi\left[\Omega_{i}\right](\sigma)=\Omega_{i}(\sigma)
$$

for all $\sigma \in \Sigma_{\infty}$.

Proof. Let $\sigma_{u} \in \Sigma$ be such that $\Omega_{i}\left(\sigma_{u}\right)=\sup \left\{\Omega_{i}(\sigma): \sigma \in \Sigma_{\infty}\right\}$. This exists since operator $\Omega$ is bounded on $\Sigma_{\infty}$.

If $\sigma_{u} \in \Sigma_{\infty}$ then $\Xi\left[\Omega_{i}\right]\left(\sigma_{u}\right)=\Omega_{i}\left(\sigma_{u}\right)$ as $\Omega_{i}(\sigma) \leq z$ implies $\Xi\left[\Omega_{i}\right](\sigma) \leq z$. By ergodicity, all continuations $\sigma^{\prime}=\left\{s^{\prime}, f_{k}\left(\sigma_{u}\right)\right\}$ satisfy $\Omega_{i}\left(\sigma_{u}\right) \geq \Omega_{i}\left(\sigma^{\prime}\right)$. Therefore $\Xi\left[\Omega_{i}\right]\left(\sigma_{u}\right)=\Omega_{i}\left(\sigma_{u}\right)$ implies $\Omega_{i}\left(\sigma_{u}\right)=\Omega_{i}\left(\sigma^{\prime}\right)$ for all continuations $\sigma^{\prime}=\left\{s^{\prime}, f_{k}\left(\sigma_{u}\right)\right\}$. Hence for any $\sigma \in \hat{\Sigma}=\left\{\sigma \in \Sigma_{\infty}: \Omega_{i}(\sigma)=\Omega_{i}\left(\sigma_{u}\right)\right\}$, all $\sigma^{\prime}=\left\{s^{\prime}, f_{k}(\sigma)\right\} \in \hat{\Sigma}$ and thus $\hat{\Sigma}=\Sigma \infty$. It follows that $\Xi\left[\Omega_{i}\right](\sigma)=\Omega_{i}(\sigma)$ for all $\sigma \in \Sigma_{\infty}$.

If $\sigma_{u} \notin \Sigma_{\infty}$ note that $\Xi\left[\Omega_{i}\right]\left(\sigma_{u}\right) \geq \Omega_{i}\left(\sigma_{u}\right)$ by the continuity of $\Omega_{i}$ and $\Xi$. If $\Xi\left[\Omega_{i}\right]\left(\sigma_{u}\right)>\Omega_{i}\left(\sigma_{u}\right)$ then there exist a continuation $\hat{\sigma}^{\prime}=\left\{\hat{s}, f_{k}\left(\sigma_{u}\right)\right\}$ with $\Omega_{i}\left(\hat{\sigma}^{\prime}\right)>\Omega_{i}\left(\sigma_{u}\right)$. Consider a sequence $\left\{\sigma_{n}\right\}$ in $\Sigma_{\infty}$ such that $\sigma_{n} \rightarrow \sigma_{u}$ and construct the sequence $\left\{\hat{\sigma}_{n}\right\} \in \Sigma_{\infty}$ given by $\hat{\sigma}_{n+1}=\left\{\hat{s}, f_{k}\left(\sigma_{n}\right)\right\}$. Since $\Omega_{i}\left(\hat{\sigma}_{n}\right) \rightarrow \Omega_{i}\left(\hat{\sigma}^{\prime}\right)$ there exists $m$ such that $\Omega_{i}\left(\hat{\sigma}_{m}\right)>\Omega_{i}\left(\sigma_{u}\right)$ contradicting $\hat{\sigma}_{m} \in \Sigma_{\infty}$. Hence $\Xi\left[\Omega_{i}\right]\left(\sigma_{u}\right)=\Omega_{i}\left(\sigma_{u}\right)$ and the reasoning for case $\sigma_{u} \in \Sigma_{\infty}$ applies as well. This proves the first statement. The proof of the second statement applies the same argument, using $\inf \left\{\Omega_{i}(\sigma): \sigma \in \Sigma_{\infty}\right\}$ instead.

Finally, let

$$
\Delta_{i}^{-}\left(s^{t}\right)=\min \left\{0, \Delta_{i}\left(s^{t}\right)\right\}
$$

and

$$
\Delta_{i}^{+}\left(s^{t}\right)=\max \left\{0, \Delta_{i}\left(s^{t}\right)\right\}
$$

Consider first

$$
\alpha^{-}=\lim _{t \rightarrow \infty} \sum_{s^{t}} \pi\left(s^{t}\right) \Delta_{i}^{-}\left(s^{t}\right)
$$

Since $\Delta_{i}^{-}\left(s^{t}\right)$ can be expressed as a continuous function of allocations on a compact support, the limit exists by Assumption 8. If

$$
\lim _{t \rightarrow \infty} \pi\left(s^{t} \in \varpi_{t}\right)=1
$$

then

$$
\alpha^{-}=\lim _{t \rightarrow \infty} \sum_{s^{t} \in \varpi_{t}} \pi\left(s^{t}\right) \Delta_{i}^{-}\left(s^{t}\right) .
$$

If $\alpha^{-}=0$ then $\Xi\left[\Omega_{i}\right](\sigma) \geq \Omega_{i}(\sigma)$ for all $\sigma \in \Sigma_{\infty}$. By Lemma 1 then $\Xi\left[\Omega_{i}\right](\sigma)=\Omega_{i}(\sigma)$ for all $\sigma \in \Sigma_{\infty}$ and

$$
\lim _{t \rightarrow \infty} \sum_{s^{t}} \pi\left(s^{t}\right) \Delta_{i}\left(s^{t}\right)=0 .
$$

The same argument applies for

$$
\alpha^{+}=\lim _{t \rightarrow \infty} \sum_{s^{t} \in \varpi_{t}} \pi\left(s^{t}\right) \Delta_{i}^{+}\left(s^{t}\right)
$$


using now the second part of Lemma 1. Hence either

$$
\lim _{t \rightarrow \infty} \sum_{s^{t}} \pi\left(s^{t}\right) \Delta_{i}\left(s^{t}\right)=0
$$

or

$$
\begin{aligned}
& \lim _{t \rightarrow \infty} \sum_{s^{t} \in \varpi_{t}} \pi\left(s^{t}\right) \Delta_{i}^{+}\left(s^{t}\right)>0, \\
& \lim _{t \rightarrow \infty} \sum_{s^{t} \in \varpi_{t}} \pi\left(s^{t}\right) \Delta_{i}^{-}\left(s^{t}\right)>0,
\end{aligned}
$$

which proves the result.

\section{A.2 Proof of Corollary 1}

If $\Delta_{i}\left(s^{t}\right)>0$ for all $s^{t} \in S^{t}$ then clearly

$$
\lim _{t \rightarrow \infty} \sum_{s^{t} \in \varpi_{t}} \pi\left(s^{t}\right) \Delta_{i}^{-}\left(s^{t}\right)=0
$$

and thus

$$
\lim _{t \rightarrow \infty} \sum_{s^{t}} \pi\left(s^{t}\right) \Delta_{i}\left(s^{t}\right)=0 .
$$

The same reasoning applies if $\Delta_{i}\left(s^{t}\right)<0$ for all $s^{t} \in S^{t}$.

\section{B Proofs for Stage 2}

\section{B.1 Proof of Proposition 2}

Condition 1 implies that there exists an allocation $\tilde{\varphi}$ and a plan for the auxiliary variable $\tilde{a} \in A^{\infty}$ such that:

$$
H_{m, i}\left(\tilde{\psi}, s^{t}\right) \leq \tilde{a}_{i m}\left(s^{t}\right)
$$

and:

$$
\tilde{a}\left(s^{t+1}\right) \in \Gamma\left(\tilde{a}\left(s^{t}\right), s_{t}\right),
$$

for all $s^{t} \in S^{t}$ for $t \geq 0, i \in I, m \in M$ and all $\tilde{a}_{0} \in A$, with the property that the sequence of probability measures $\left\{\tilde{\mu}_{t}\right\}$ on $K \times X$ associated with $\tilde{\psi}$ converges weakly to $\mu_{\infty}^{f b}$.

Define $\bar{a}_{t}=\max _{s^{t} \in S^{t}}\left\{H\left(\tilde{\psi}, s^{t}\right)\right\}$ for all $t \geq 0$ and $\hat{a}_{t}=\max \left\{\bar{a}_{t}, \hat{a}_{t-1}\right\}$ for all $t \geq 1$, where the maximum is taken componentwise and $\hat{a}_{0}=\tilde{a_{0}}$. Since $\tilde{\psi}$ is admissible, each element in the sequence $\left\{\widehat{a}_{t}\right\}$ is in $A$. By construction, the sequence $\left\{\hat{a}_{t}\right\}$ is monotone increasing. Since $A$ is a compact set, $\lim _{t \rightarrow \infty} \hat{a}_{t}=\hat{a}_{\infty} \in A$. By weak convergence of $\left\{\tilde{\mu}_{t}\right\}$, and the continuity of $H$ in Assumption 4, we have 
that

$$
\lim _{t \rightarrow \infty_{s^{j} \in S^{j}, j \geq t}} \sup _{t}\left\{H_{m, i}\left(\tilde{\psi}, s^{j}\right)\right\}=\lim _{t \rightarrow \infty_{s^{j} \in S^{j}, j \geq t}} \sup _{m, i}\left\{H_{m}\left(\psi^{f b}, s^{j}\right)\right\} \leq \hat{a}_{\infty},
$$

for all $i \in I, m \in M$.

\section{B.2 Proof of Proposition 3}

If $\alpha \in A^{f b}$ the result is trivial. If $\alpha \in \operatorname{int}(\Gamma(\alpha, s))$ for some $s \in S$, then the result follows from Assumption 5. Consider thus $\alpha \notin A^{f b}$ and $\alpha \notin i n t(\Gamma(\alpha, s))$ for all $s$. By Assumption $5, \alpha \in \Gamma(\alpha, s)$ and thus $\alpha$ is an adjacent point to $\Gamma(\alpha, s)$. Since the image of $\Gamma(\alpha, s)$ is a convex set by Assumption 5 , we apply the Separating Hyperplane Theorem to find a half-space $\chi_{s}=\{\gamma \in A: p \gamma \geq p \alpha \in \Re\}$ with $p \neq \overrightarrow{0}$, such that $\Gamma(\alpha, s) \subset \chi_{s}$ for a given $s \in S$.

We now show that for some $s \in S, A^{f b} \cap \chi_{s} \neq \varnothing$ for any half-space $\chi_{s}$. We proceed by contradiction. Assume that for every $s \in S$ there exists a half-space $\chi_{s}$ such that $A^{f b} \cap \chi_{s}=\varnothing$. Condition 1 implies that the set $A^{f b}$ is non-empty by Proposition 2 and that for some $s^{*} \in S$, there exists $x \in \chi_{s^{*}}$ with $y \in \Gamma\left(x, s^{*}\right), y \notin \chi_{s^{*}}$. Otherwise there would be no admissible plan with $a_{0} \in \chi_{s^{*}}$ that would converge to $A^{f b}$.

Let $w \in A$ satisfy $\alpha=\varrho w+(1-\varrho) x$ for some $\varrho \in(0,1)$. Such a point will belong to the closure of the complement of $\chi_{s^{*}}$, the set $\{\gamma \in A: p \gamma \leq p \alpha \in \Re\}$. Since $w \in \Gamma\left(w, s^{*}\right)$, the convexity of $\Gamma$ implies that $\varrho y+(1-\varrho) w \in \Gamma\left(\alpha, s^{*}\right)$ but $\varrho y+(1-\varrho) w \notin \chi_{s^{*}}$. Thus, we reach a contradiction and therefore $A^{f b} \cap \chi_{s} \neq \varnothing$ for some $s \in S$.

Finally, consider the set:

$$
G=\left\{(1-\varrho) \alpha+\varrho \alpha^{\prime} \in A: \varrho \in(0,1], \alpha^{\prime} \in A^{f b}\right\}
$$

This is a convex set with $A^{f b} \subset G$. If $\Gamma(\alpha, s) \cap G=\varnothing$ for all $s \in S$, it would then be possible to find a separating hyperplane $\chi_{s}$ for each $s$ with $\Gamma(\alpha, s) \subset \chi_{s}$ and $G \cap \chi_{s} \neq \varnothing$. This would contradict our previous result. Thus for some $s \in S, \Gamma(\alpha, s) \cap G \neq \varnothing$ and the result follows.

\section{B.3 Proof of Proposition 4}

Let $\psi^{*}$ be a second best plan and assume that Condition 1 holds. We prove the result by constructing an auxiliary variable plan $a^{*}$ such that $\left\{\psi^{*}, a^{*}\right\}$ is admissible and $\lim _{t \rightarrow \infty} \pi\left(s^{t} \in \varpi_{t}\right)=1$. We start with two Lemmas that will be useful to characterize the choice of $a^{*}$.

Lemma 2 Let a be an admissible plan for the auxiliary variable with a $\left(s^{t}\right) \in \operatorname{int}\left(\Gamma\left(a\left(s^{t-1}\right), s_{t}\right)\right)$ for some $s^{t} \in S^{t}$. Then there exists an admissible plan $\tilde{a}$ with $\tilde{a}\left(s^{t}\right)>a\left(s^{t}\right)$ and $\tilde{a}\left(s^{j}\right) \geq a\left(s^{j}\right)$ for all $s^{j} \in S^{j} \mid s^{t}$; and $\tilde{a}\left(s^{j}\right)=a\left(s^{j}\right)$ elsewhere. Moreover if $a\left(s^{j}\right) \in \operatorname{int}(A)$ for all $s^{j} \in S^{j} \mid s^{t}$ then $\tilde{a}$ can be found such that $\tilde{a}\left(s^{j}\right)>a\left(s^{j}\right)$ for all $s^{j} \in S^{j} \mid s^{t}$.

Proof. We prove the result by construction. Let $a\left(s^{t}\right) \in \operatorname{int}\left(\Gamma\left(a\left(s^{t-1}\right), s_{t}\right)\right)$. Let $\tilde{a}=a$ everywhere but in the set $\left\{S^{j} \mid s^{t}: j \geq t\right\}$. Let $\gamma=[\bar{a}]_{m, i} \in \Re^{I M}$ be the join for $A$. Since $a\left(s^{t}\right) \in$ 
$\operatorname{int}\left(\Gamma\left(a\left(s^{t-1}\right), s_{t-1}\right)\right), a\left(s^{t}\right) \in \operatorname{int}(A)$ and thus $a\left(s^{t}\right)<\gamma$. Then, for sufficiently small scalar $\varrho>0$ :

$$
\tilde{a}\left(s^{t}\right)=\varrho \gamma+(1-\varrho) a\left(s^{t}\right) \in \Gamma\left(a\left(s^{t-1}\right), s_{t}\right)
$$

with $a\left(s^{t}\right)<\tilde{a}\left(s^{t}\right)$. By Assumption 5, $\Gamma$ is convex and $\gamma \in \Gamma(\gamma, s)$, and thus

$$
\tilde{a}\left(s^{t+1}\right)=\varrho \gamma+(1-\varrho) a\left(s^{t+1}\right) \in \Gamma\left(\tilde{a}\left(s^{t}\right), s_{t}\right) .
$$

Construct $\tilde{a}$ for all $s^{j} \in S^{j} \mid s^{t}, j \geq t+1$ by iteration. The plan $\tilde{a}$ is admissible and $\tilde{a}\left(s^{j}\right) \geq a\left(s^{j}\right)$ for all $s^{j} \in S^{j} \mid s^{t}$ by the definition of $\gamma$ and $\varrho>0$. The corollary follows trivially

The second Lemma uses the quasi-concavity of $W$.

Lemma 3 Let $\{\psi, a\}$ be admissible, with $U\left(x, s_{0}\right)=W(a)$. Then for all admissible $a^{\prime}$ such that $a^{\prime} \geq a$ (component-wise), we have $W\left(a^{\prime}\right) \geq W(a)$. Moreover, if $s^{t} \notin \varpi_{t}$ and $a^{\prime}\left(s^{j}\right)>a\left(s^{j}\right)$ for all $s^{j} \in S^{j} \mid s^{t}$ then either $W\left(a^{\prime}\right)>W(a)$ or there exists an admissible pair $\left\{\psi^{\prime}, a^{\prime}\right\}$ such that $U\left(x^{\prime}, s_{0}\right)=W\left(a^{\prime}\right)$ and $s^{t} \in \varpi_{t}^{\prime}$.

Proof. The first part is trivial as $\left\{\psi, a^{\prime}\right\}$ is admissible so $W\left(a^{\prime}\right)$ cannot be worse than $W(a)$. Let $s^{t} \notin \varpi_{t}$ and $a^{\prime}\left(s^{t}\right)>a\left(s^{t}\right)$. If $W\left(a^{\prime}\right)=W(a)$ then $U\left(x, s_{0}\right)=W\left(a^{\prime}\right)$ so setting $\psi^{\prime}=\psi$ we have $s^{t} \in \varpi_{t}^{\prime}$

Since $\psi^{*}$ is admissible, there exists at least one auxiliary variable plan $a$ such that $\left\{\psi^{*}, a\right\}$ is admissible. The sequence $\left\{\pi\left(s^{t} \in \varpi_{t}\right)\right\}_{t}$ is weakly increasing and every element belongs to [0,1], hence it converges. If $\lim _{t \rightarrow \infty} \pi\left(s^{t} \in \varpi_{t}\right)=1$ the result is proved by equating $a$ with $a^{*}$.

If $\lim _{t \rightarrow \infty} \pi\left(s^{t} \in \varpi_{t}\right)<1$, let $\tilde{S}^{\infty} \subseteq S^{\infty}$ be the subset of histories such that

$$
s^{t} \notin \varpi_{t}
$$

for all $t \geq 0$. If $a\left(s^{t}\right) \in A^{f b}$ for any $t$ and $s^{\infty} \in \tilde{S}^{\infty}$, then by Assumption 5 there exists an admissible auxiliary variable plan $a^{\prime}$ with $a^{\prime}\left(s^{j}\right)=a\left(s^{t}\right)$ for all $s^{j} \in S^{j} \mid s^{t}, a^{\prime}=a$ elsewhere. Clearly $a^{\prime}\left(s^{j}\right) \in A^{f b}$ for all $s^{j} \in S^{j} \mid s^{t}$ and thus by Assumption 7 we have that $s^{t} \in \varpi_{t}^{\prime}$ and $\left\{\psi^{*}, a^{\prime}\right\}$ is admissible. By induction either we find an admissible $a^{\prime}$ with $\lim _{t \rightarrow \infty} \pi\left(s^{t} \in \varpi_{t}^{\prime}\right)=1$, which proves the result; or for some plan $a$ with $\left\{\psi^{*}, a\right\}$ admissible we have that $a\left(s^{t}\right) \notin A^{f b}$ for all $s^{t} \subset s^{\infty} \in \tilde{S}^{\infty}$.

Consider now the possibility that for plan $a$ we have $a\left(s^{t}\right) \in \operatorname{int}\left(\Gamma\left(a\left(s^{t-1}\right), s_{t}\right)\right)$ for some $s^{t} \in \tilde{S}^{\infty}$. If $a\left(s^{j}\right) \in \operatorname{int}(A)$ for all $s^{j} \in S^{j} \mid s^{t}$ then by Lemma 2 there exists another admissible $a^{\prime}$ with $a^{\prime}\left(s^{t}\right)>a\left(s^{t}\right)$ (component-wise) for all $s^{j} \in S^{j} \mid s^{t}, a^{\prime}=a$ elsewhere. By Lemma 3 we have that $s^{t} \in \varpi_{t}^{\prime}$ as $W(a)<W\left(a^{\prime}\right)$ would contradict $\psi^{*}$ being the second best. Lemma 2 still applies if $a\left(s^{j}\right) \notin \operatorname{int}(A)$ because $a_{m, i}\left(s^{j}\right)=0$ for some $m, i$. If $a\left(s^{j}\right) \notin \operatorname{int}(A)$ because $a_{m, i}\left(s^{j}\right)=\bar{a}$ for some $m, i$ then by Assumption 5 we can work with the subspace $\bar{A}=\left\{\alpha \in A: \alpha_{m, i}=\bar{a}\right\}$ for all $s^{d} \in S^{d} \mid s^{j}$. By Proposition 2, $A^{f b} \neq \varnothing$ and follows that the vector $\gamma=[\bar{a}]_{m, i}$ (the join 
of $A$ ) must belong to $A^{f b}$. Since $a\left(s^{j}\right) \notin A^{f b}, a\left(s^{j}\right) \neq \gamma$ and $\bar{A}$ is not a singleton. Defining $\operatorname{int}\left(\Gamma\left(a\left(s^{t-1}\right), s_{t}\right)\right)$ on $\bar{A}$ we can apply Lemma 2 again. By induction either we find a plan $a^{\prime}$ such that $\left\{\psi^{*}, a^{\prime}\right\}$ is admissible with $\lim _{t \rightarrow \infty} \pi\left(s^{t} \in \varpi_{t}^{\prime}\right)=1$, which proves the result; or for some admissible plan $a$ we have that $a\left(s^{t}\right) \notin A^{f b}$ and $a\left(s^{t}\right) \notin \operatorname{int}\left(\Gamma\left(a\left(s^{t-1}\right), s_{t}\right)\right)$ for all $s^{t} \subset s^{\infty} \in \tilde{S}^{\infty}$.

It follows that we find $a$ such that $\left\{\psi^{*}, a\right\}$ is admissible and satisfies either (i) $\lim _{t \rightarrow \infty} \pi\left(s^{t} \in \varpi_{t}\right)=$ 1 or (ii) $a\left(s^{t}\right) \notin A^{f b}, a\left(s^{t}\right) \notin \operatorname{int}\left(\Gamma\left(a\left(s^{t-1}\right), s_{t}\right)\right)$ for all $s^{t} \subset s^{\infty} \in \tilde{S}^{\infty}$. Let $a$ be an admissible plan that satisfies (ii) and define:

$$
B(\alpha)=\left\{\alpha^{\prime} \in A: \alpha^{\prime}>\alpha\right\}
$$

where the inequality is taken component-wise.

Lemma 4 Let $\left\{\psi^{*}, a\right\}$ be an admissible plan. For all $s^{t-1} \in S^{t-1}$ such that $a\left(s^{t-1}\right) \notin A^{f b}$, there exists $s_{t}$ with

$$
\Gamma\left(a\left(s^{t-1}\right), s_{t}\right) \cap B\left(a\left(s^{t-1}\right)\right) \neq \varnothing .
$$

Proof. By Proposition 3 there exists one $s_{t}$ and $\varrho>0$, such that $\varrho a\left(s^{t-1}\right)+(1-\varrho) \alpha \in$ $\operatorname{int}\left(\Gamma\left(a\left(s^{t-1}\right), s_{t}\right)\right)$ for $\alpha \in A^{f b}$. By Assumption 7 and the definition of $\varpi_{t}$ and $A^{f b}, a\left(s^{t-1}\right)<A^{f b}$ if $a\left(s^{t-1}\right) \notin A^{f b}$ and thus $\Gamma\left(a\left(s^{t-1}\right), s_{t}\right) \cap B\left(a\left(s^{t-1}\right)\right) \neq \varnothing$

Let $s^{t} \subset s^{\infty} \in \tilde{S}^{\infty}$ for state $s_{t}$ such that $\Gamma\left(a\left(s^{t-1}\right), s_{t}\right) \cap B\left(a\left(s^{t-1}\right)\right) \neq \varnothing$. Since $a\left(s^{t}\right) \notin$ $\operatorname{int}\left(\Gamma\left(a\left(s^{t-1}\right), s_{t}\right)\right)$, either $a\left(s^{t}\right) \in B\left(a\left(s^{t-1}\right)\right)$ or there exists $a^{\prime}\left(s^{t}\right) \in B\left(a\left(s^{t-1}\right)\right)$ with $a^{\prime}\left(s^{t}\right)>$ $a\left(s^{t}\right)$ component-wise. For the latter, the steps in Lemma 2 and the previous argument show that there exists $a^{\prime}$ such that $\left\{\psi^{*}, a^{\prime}\right\}$ is admissible and $s^{t} \in \varpi_{t}^{\prime}$. Thus we continue with $a\left(s^{t}\right) \in$ $B\left(a\left(s^{t-1}\right)\right)$ for all states.

Let $s^{t} \subset s^{\infty} \in \tilde{S}^{\infty}$ for state $s_{t}$ such that $\Gamma\left(a\left(s^{t-1}\right), s_{t}\right) \cap B\left(a\left(s^{t-1}\right)\right) \neq \varnothing$. If such event is recurrent, that is, for any $d$ there exists $t \geq d$ such that $\Gamma\left(a\left(s^{t-1}\right), s_{t}\right) \cap B\left(a\left(s^{t-1}\right)\right) \neq \varnothing$ then $a$ that converges to $\gamma=[\bar{a}]_{m, i}$. We use here the fact that the second best is proper, so $A^{f b} \neq \gamma$. Thus there exists a finite $d$ such that $a\left(s^{d}\right) \in A^{f b}$ and we can apply the previous argument.

Finally we show that the set of histories $s^{\infty} \in \tilde{S}^{\infty}$ such that $\Gamma\left(a\left(s^{t-1}\right), s_{t}\right) \cap B\left(a\left(s^{t-1}\right)\right) \neq \varnothing$ is not recurrent has measure zero. For a given node $s^{d} \subset s^{\infty} \in \tilde{S}^{\infty}$, let $P_{j}$ for $j \geq d$ be the probability that event $\Gamma\left(a\left(s^{t-1}\right), s_{t}\right) \cap B\left(a\left(s^{t-1}\right)\right) \neq \varnothing$ has occurred for some $t \leq j$ and $s^{t} \in S^{t} \mid s^{d}$. Since $s^{d} \subset s^{\infty} \in \tilde{S}^{\infty}, a\left(s^{t-1}\right) \notin A^{f b}$ and by Lemma 4 there exists at least one $s_{t}$ such that $\Gamma\left(a\left(s^{t-1}\right), s_{t}\right) \cap B\left(a\left(s^{t-1}\right)\right) \neq \varnothing$ occurs. By Assumption $1, \pi\left(s_{t}\right) \geq \delta>0$, so

$$
P_{t} \geq P_{t-1}+\delta\left(1-P_{t-1}\right)
$$

Clearly $P_{t}$ converges to 1 and thus the set of histories such that $\Gamma\left(a\left(s^{t-1}\right), s_{t}\right) \cap B\left(a\left(s^{t-1}\right)\right) \neq \varnothing$ is not recurrent has measure zero. It follows that $\lim _{t \rightarrow \infty} \pi\left(s^{t} \in \varpi_{t}\right)=1$. 